\title{
THE DYNAMICS OF THE MERGING GALAXY CLUSTER SYSTEM A2256: EVIDENCE FOR A NEW SUBCLUSTER
}

\author{
ROBERT C. BERRINGTON ${ }^{1}$ \\ Space Science Division, Code 7653, Naval Research Laboratory, 4555 Overlook Avenue, Washington, DC 20375-5352; rberring@gamma.nrl.navy.mil \\ AND \\ Phyllis M. Lugger and Haldan N. Cohn \\ Department of Astronomy, Indiana University, 727 East Third Street, Swain Hall West 319, Bloomington, IN 47405-7105; \\ lugger@astro.indiana.edu,cohn@astro.indiana.edu \\ Received 1999 July 27; accepted 2002 January 22
}

\begin{abstract}
We present 236 new radial velocities of galaxies in the cluster A2256 measured with the WIYN Hydra Multi-Object Spectrograph. Combined with the previous work of Fabricant, Kent, \& Kurtz, we have velocities for a total of 319 galaxies, of which 277 are cluster members. In addition to the new radial velocities, we present a $3 \times 3$ image mosaic in the $R$ band of the central $19^{\prime} \times 19^{\prime}$ region of A2256, from which we obtained photometry for 861 galaxies. These data provide strong evidence for a merger event between two groups. In addition, we present evidence for the presence of a third group, on the outer reaches of the system, that is just now beginning to merge with the system.
\end{abstract}

Key words: galaxies: clusters: individual (A2256)

\section{INTRODUCTION}

Originally thought to be a highly relaxed or evolved cluster, A2256 was shown by Dressler (1978) to display evidence for equipartition in energy. With a richness class 2, A2256 is surpassed only by $5 \%$ of the clusters found in Abell's (1958) catalog. Faber \& Dressler (1977) measured the velocity dispersion to be $1351 \mathrm{~km} \mathrm{~s}^{-1}$, making it one of the highest velocity dispersions of known galaxy clusters. Carter \& Metcalfe (1980) showed the galaxy distribution to be elliptical. Fabricant, Kent, \& Kurtz (1989) increased the number of measured radial velocities to 89 and found a flat velocity histogram. They first suggested the presence of substructure and interpreted this as indicating that A2256 represents a merger in progress.

In the X-ray, A2256 is a luminous and hot cluster with a central temperature of $\sim 7 \mathrm{keV}$. First imaged by the Einstein IPC, the X-ray emission from A2256 was found to depart significantly from spherical symmetry (Fabricant, Rybicki, \& Gorenstein 1984). Observations by ROSAT resolved the nonspherical X-ray emission into two maxima in the central region of the cluster (Briel et al. 1991). Briel \& Henry (1994) used several Position Sensitive Proportional Counter (PSPC) observations to obtain a temperature map of A2256. They found an extremely complicated temperature structure with two opposing $12.0 \mathrm{keV}$ lobes almost perpendicular to a proposed infall direction for the two merging subgroups. However, the uncertainties of this temperature map are large. Later observations by $A S C A$ did not observe a similar central temperature structure but did find a steep radial temperature profile in agreement with ROSAT data (Markevitch 1996).

A2256 also exhibits a number of striking features in the radio regime, as discussed by Röttgering et al. (1994). It has at least four galaxies with head-tail morphologies, placing it low.

${ }^{1}$ American Society for Engineering Education (ASEE) Posdoctoral Fel- among three to four other clusters with comparable numbers of confirmed head-tail radio features. It also contains one of the longest and narrowest head-tail structures at 700 $\mathrm{kpc}$ in length and less than $2.5 \mathrm{kpc}$ in width. A Z-shaped structure with an ultrasteep spectrum has been tentatively identified with a cluster galaxy, but it eludes definitive identification. The most pronounced radio features in A2256 are two extended halos with spatial dimensions that are estimated at $1.0 \times 0.3 \mathrm{Mpc}$.

A2256 is well suited for the study of substructure and the role of dominant galaxies. Its optical structure is very much like that of the Coma Cluster (A1655) with a central pair of dominant galaxies, each at the center of a concentration of fainter galaxies. The galaxy distribution also indicates the likely presence of the substructure that is seen in the X-ray images. In this paper we present new optical imaging of the central region of the cluster and 278 new radial velocities obtained with the Wisconsin-Indiana-YaleNOAO (WIYN) telescope. In $\S 2$ we describe both new imaging and spectroscopy obtained by the WIYN telescope. We also include the results of the data analysis. In $\S 3$, we review the statistical methods for searching for substructure. In $\S 4$ we include a discussion of the implications of the observations and the statistical tests. In $\S 5$ we summarize the results of this study.

\section{DATA}

\subsection{CCD Imaging Observations}

Since previous study of the galaxy distribution in A2256 has been based on scans of Palomar Observatory Sky Survey plates (Fabricant et al. 1989), we obtained deep CCD images of the central region of A2256. We used the WIYN $3.5 \mathrm{~m}$ telescope at the Kitt Peak National Observatory (KPNO) to acquire a total of 18 images in the form of a $3 \times 3$ mosaic centered on the cluster's center of $\alpha=17^{\mathrm{h}} 4^{\mathrm{m}} 11^{\mathrm{s}} .1, \delta=78^{\circ} 38^{\prime} 3^{\prime \prime} 6(\mathrm{~J} 2000.0)$. At the time of these 
TABLE 1

Field CENTERS

\begin{tabular}{cccc}
\hline \hline $\begin{array}{c}\text { Field } \\
(1)\end{array}$ & $\begin{array}{c}\text { R.A. } \\
(\mathrm{J} 2000.0) \\
(2)\end{array}$ & $\begin{array}{c}\text { Decl. } \\
(\mathrm{J} 2000.0) \\
(3)\end{array}$ & $\begin{array}{c}\text { Run } \\
(4)\end{array}$ \\
\hline C3 $\ldots \ldots .$. & 170607.5 & 784425.0 & 1996 Jun \\
C4 ..... & 170608.3 & 783825.0 & 1996 Jun \\
C5 ..... & 170558.0 & 783250.7 & 1997 Jul \\
D3..... & 170352.0 & 784411.0 & 1996 Jun \\
D4..... & 170401.8 & 783811.4 & 1996 Jun \\
D5..... & 170404.2 & 783141.7 & 1997 Jul \\
E3 ...... & 170141.0 & 784400.0 & 1996 Jun \\
E4 ..... & 170145.4 & 783729.0 & 1996 Jun \\
E5 ...... & 170140.0 & 783240.0 & 1996 Jun \\
\hline
\end{tabular}

Note.-Units of right ascension are hours, minutes, and seconds, and units of declination are degrees, arcminutes, and arcseconds.

observations, the WIYN imager used a $2 \mathrm{k} \times 2 \mathrm{k}$ Tektronix CCD to image a 6.7 square field at a scale of 0.20 pixel $^{-1}$.

We obtained two $600 \mathrm{~s}$ Kron-Cousins $R$-band $\left(R_{\mathrm{KC}}\right)$ exposures per field. The fields are labeled east to west by $\mathrm{C}$, $\mathrm{D}$, and $\mathrm{E}$ and north to south by 3,4 , and 5 . The central field D4 of the mosaic is centered on the cluster center. The surrounding fields were offset by steps of 6.2 in R.A. and decl., resulting in a 100 pixel overlap in fields. However, a small portion of the sky was missed between images D5 and E5 as a result of an error in telescope pointing. Otherwise, coverage was complete over the entire mosaic. Table 1 shows the R.A. and decl. of the center of each image frame, as well as the observation date.

We used the IMRED.CCDRED package in $\mathrm{IRAF}^{2}$ to reduce the images. All images were bias subtracted, flat fielded, and dark current subtracted. In addition, each frame contains a dead column and a hot column that were removed using the FIXPIX procedure. Each frame pair was co-added by the IMCOMBINE procedure after being matched, translated, and rotated by the GEOMAP/GEOTRAN procedures. Most images required a shift of less than a pixel. However, a few images were taken with an appreciable difference in time between the two pair members and thus required shifts on the order of 50-100 pixels.

To find galaxy positions, we used the Faint Object Classification and Analysis System (FOCAS) to locate and produce positions for all the galaxies within each frame. To perform the astrometry, we obtained a list of approximately 300 stars within the $3 \times 3$ WIYN mosaic from the Digital Sky Survey (DSS) Quick-V scans. Any star found to be saturated on a WIYN image was removed from the list. The unsaturated stars were centroided on each WIYN frame and used to find a plate solution, based on at least 20 stars.

We used FOCAS to perform the photometry on each WIYN frame. Instrumental magnitudes were obtained from all the fields and transformed into the $R_{\mathrm{KC}}$ band. To correct for Galactic extinction, we adopt the relation

$$
A_{R_{\mathrm{KC}}}=2.47 E(B-V),
$$

\footnotetext{
${ }^{2}$ IRAF is distributed by the National Optical Astronomy Observatories, which are operated by the Association of Universities for Research in Astronomy, Inc., under cooperative agreement with the National Science Foundation.
}

which was obtained from the transformations of Fernie (1983). Values of $E(B-V)$ were obtained from the color excess maps of Burstein \& Heiles (1982). $K$-corrections conform to the procedures described by Postman \& Lauer (1995) and Pence (1976).

From the $3 \times 3$ WIYN image mosaic, we obtained a cata$\log$ of 861 galaxies. All matches from overlapping CCD frames were removed to prevent any redundancy. Because the astrometry was done using a different set of stars for each frame, all the matches were extracted and their positions were checked against each other to estimate the accuracy of the astrometry. A total of 30 matching pairs were found, and the difference in positions was calculated. Astrometry of the matching pairs is susceptible to a number of errors. The plate solution is worst at the edge of the plate since the distribution of the stars used in finding the plate solution may not fully cover the CCD from edge to edge. Because galaxies are extended objects, portions of galaxies near the edge of a WIYN frame are sometimes missing from the image. For these galaxies, the image that contained the entire galaxy was used for both the photometric and astrometric work. By comparing the matching pairs of galaxies on adjacent WIYN frames, we estimate accuracy of the galaxy positions to be 0 ". 3 .

\subsection{Spectroscopy}

We report radial velocity measurements for 278 galaxies, of which 48 are in common with the samples measured by Fabricant et al. (1989) and Faber \& Dressler (1977). By combining these new measurements with velocities for 41 additional galaxies from these two previous studies, we have developed a sample of 319 galaxy velocities for our analysis. All new radial velocities were obtained using the Hydra Multi-Object Spectrograph on the WIYN $3.5 \mathrm{~m}$ telescope at KPNO. Both the imaging data and the spectroscopic data were taken over several runs. An observation log for the spectroscopic data is given in Table 2.

In addition to the 861 galaxy catalog obtained from the WIYN $3 \times 3$ image mosaic, we obtained an image from the DSS Quick-V scans. Using the FOCAS package, we obtained a list of galaxies within \pm 0.5 of the cluster center. The combination of this list with the catalog from the WIYN $3 \times 3$ image mosaic and the galaxy list from Fabricant et al. (1989) produced a total list of 1090 galaxies, which was used in the galaxy selection.

The Hydra spectrograph contains 250 ports within which are placed fibers that act as apertures for the spectrograph. Either of two 100 fiber cable sets may be selected, one optimized for the blue and one for the red. Galaxy selection was carried out using the WHYDRA program, which optimizes fiber placement based on a set of user-defined weights, subject to a set of fiber placement rules. Our method assigned

TABLE 2

Hydra OBSERVATION LOG

\begin{tabular}{|c|c|c|c|c|}
\hline $\begin{array}{l}\text { Observation Date } \\
\text { (1) }\end{array}$ & $\begin{array}{l}\text { Grating } \\
\text { (2) }\end{array}$ & $\begin{array}{c}\text { Central } \lambda \\
(\mathrm{nm}) \\
(3)\end{array}$ & $\begin{array}{c}\text { Fibers } \\
\text { (4) }\end{array}$ & $\begin{array}{c}\text { Exposure } \\
\text { (s) } \\
\text { (5) }\end{array}$ \\
\hline 1996 Jun ................... & 360 at 7.0 & 750.0 & Red & $3 \times 1800$ \\
\hline 1997 Jun ................... & 600 at 13.9 & 580.0 & Blue & $2 \times 3600$ \\
\hline 1998 Jun................... & 600 at 13.9 & 570.0 & Blue & $2 \times 3600$ \\
\hline
\end{tabular}


weights according to the magnitude of the galaxy and required that at least 20 fibers be placed on blank sky.

For the 1996 June run, we chose the $500-1000 \mathrm{~nm}$ band and the red cable to observe the $\mathrm{H} \alpha, \mathrm{H} \beta, \mathrm{Mg} b$, and $\mathrm{Na} \mathrm{D}$ lines. The red fiber set uses a $2^{\prime \prime}$ size fiber that is optimized for the 450-1000 nm wavelength range. Because of the presence of strong sky lines, the spectra redward of $\sim 700 \mathrm{~nm}$ were not usable for velocity measurements. Exposure sets of $3 \times 30$ minutes were used for the first two fields and $2 \times 20$ minutes for the third field. For the 1997 June and 1998 June runs, we chose a higher dispersion and the blue cable to concentrate on the 430-720 nm region. The blue fiber set uses a $3^{\prime \prime}$ size fiber. The exposure sets with the blue cable were all $2 \times 1 \mathrm{hr}$. We bracketed each set for both runs by a Th-Ar comparison lamp exposure for wavelength calibration. Each exposure was bias subtracted using the IRAF CCDRED package. All spectra were flat fielded, dispersion corrected, and extracted using the IRAF HYDRA package.

A minimum of 20 fibers were used to obtain sky spectra, which were combined to form a sky spectrum template. All galaxy spectra were then sky subtracted. The only problematic sky feature was the $\lambda \lambda 557.7 \mathrm{~nm}[\mathrm{O}$ I] line. Although it usually subtracted cleanly, it was sometimes necessary to remove it manually. All exposures for each setup were averaged together using a $3 \sigma$ cosmic-ray clip. Figure 1 shows a typical spectrum with target lines marked.

We extracted radial velocities using the IRAF FXCOR package. For both the red and blue cable setups, only the $450-720 \mathrm{~nm}$ spectral region was used in determining the radial velocities. Three templates of objects with known radial velocities were obtained for the cross-correlation. Two stellar templates, AGK 224 and HD 223094, and one galaxy template, NGC 7331, of radial velocities $-49.5,26.0$, and $819.0 \mathrm{~km} \mathrm{~s}^{-1}$, respectively, were each used to obtain the heliocentric velocity of each galaxy.

Depending on the spectrograph setup and galaxy magnitude, the uncertainties of the radial velocities varied over the range of $10-120 \mathrm{~km} \mathrm{~s}^{-1}$ with a median uncertainty of $52 \mathrm{~km}$ $\mathrm{s}^{-1}$. In the 1996 June run, a total of 31 galaxies done by Fabricant et al. (1989) were redone. Figure 2 shows a comparison of our measured velocities with those of Fabricant et al. (1989) as a function of $m_{r}$. The mean offset is $-55 \mathrm{~km} \mathrm{~s}^{-1}$, with a standard deviation of $98 \mathrm{~km} \mathrm{~s}^{-1}$ and an error of the

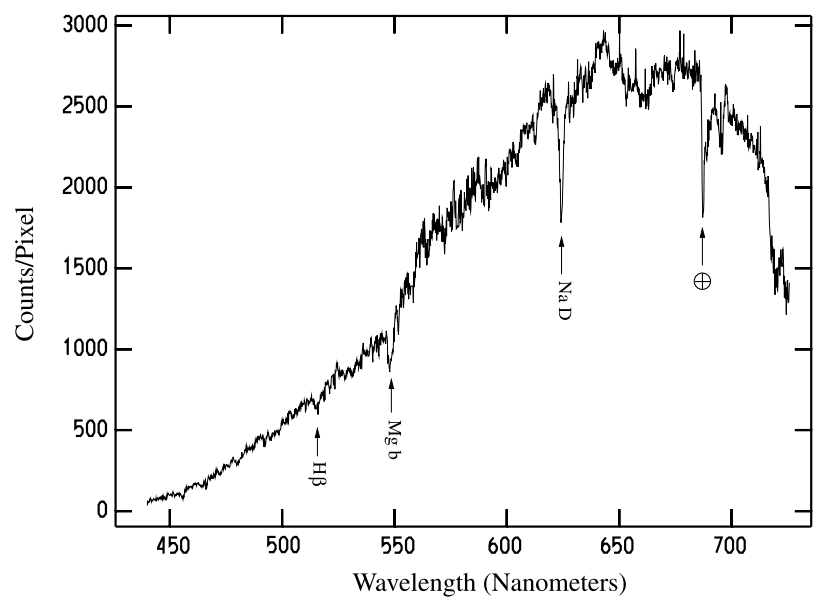

FIG. 1.-Example reduced galaxy spectrum with the target lines marked. $\mathrm{H} \alpha$ is often redshifted into the telluric feature at $680 \mathrm{~nm}$, as is the case here.

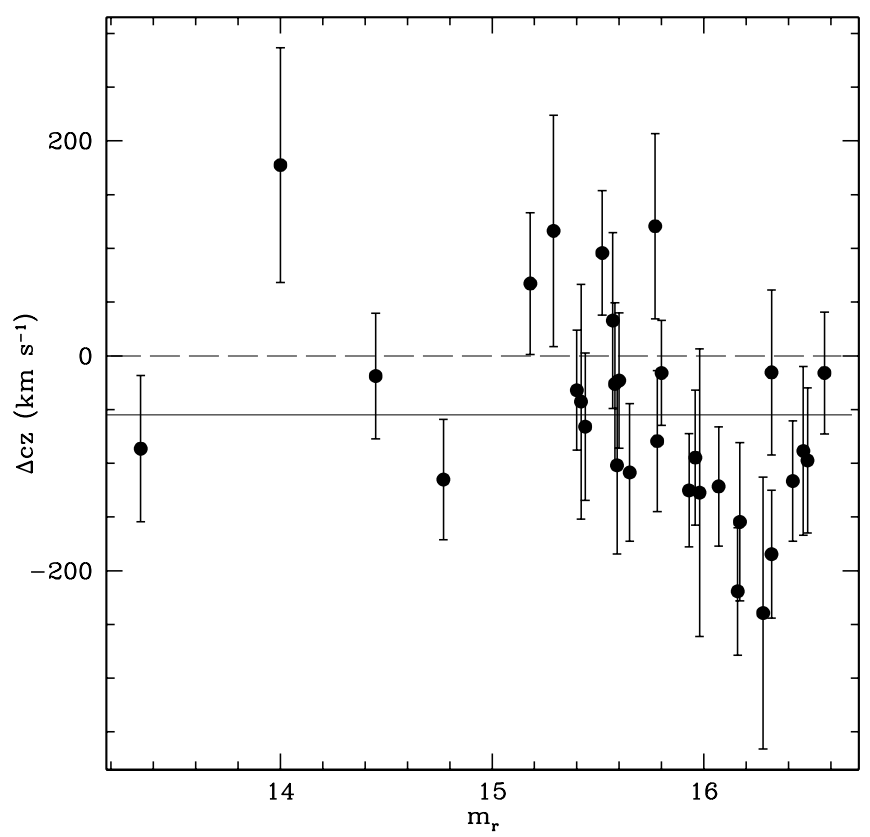

FIG. 2.-Difference between the velocities from Fabricant et al. (1989) and ours from the 1996 June run. We find a mean difference of $55 \mathrm{~km} \mathrm{~s}^{-1}$ with a standard deviation of $98 \mathrm{~km} \mathrm{~s}^{-1}$. The error bars are $1 \sigma$ error bars as calculated from the quadratic sum of the two uncertainties.

mean of $18 \mathrm{~km} \mathrm{~s}^{-1}$. While the mean offset is somewhat larger than expected, it is still small compared with the combined measurement errors for the two studies.

In addition to the velocities redone in the 1996 June run, 45 galaxies from Fabricant et al. (1989) were redone in the 1997 June and 1998 June runs. We performed a comparison of these galaxy velocities with both those that were measured in 1996 June and those of Fabricant et al. (1989). We found all the velocities measured in 1997 June and 1996 June to be consistent with each other within the $1 \sigma$ level. The offset between the values measured by Fabricant et al. (1989) and our values persisted in the 1997 June and 1998 June measurements, with a mean offset of $-70 \mathrm{~km} \mathrm{~s}^{-1}$, a standard deviation of $84 \mathrm{~km} \mathrm{~s}^{-1}$, and an error of the mean of $13 \mathrm{~km} \mathrm{~s}^{-1}$. Figure 3 shows a comparison between the Fabricant et al. (1989) and the 1996 June runs versus the magnitude $m_{r}$. We checked the wavelength of the $\lambda \lambda 557.7$ $\mathrm{nm}$ skyline to check for any instrument-dependent offsets. No such offsets were found in any of the HYDRA setups. Because our two spectrograph setups used not only different fibers but also different central wavelengths and gratings, we conclude that the offset between our values and those of Fabricant et al. (1989) most likely reflects the lower accuracy of the previous study. In any case, the offset is within the measurement errors.

All radial velocity data obtained in the WIYN observing runs described in Table 2 are reported in Table 3. For each velocity reported by the IRAF FXCOR package, a visual check between the velocity standard and the cluster galaxy was performed to avoid accidental selection of any spurious peaks in the cross-correlation. During the three nights a total of five different setups were used for A2256. In each setup, at least 20 fibers were used to obtain the sky spectra, and on average 11 fibers were used for the previously measured galaxies. The remaining $\sim 50$ fibers were used for galaxies selected from the DSS Quick-V scans. 


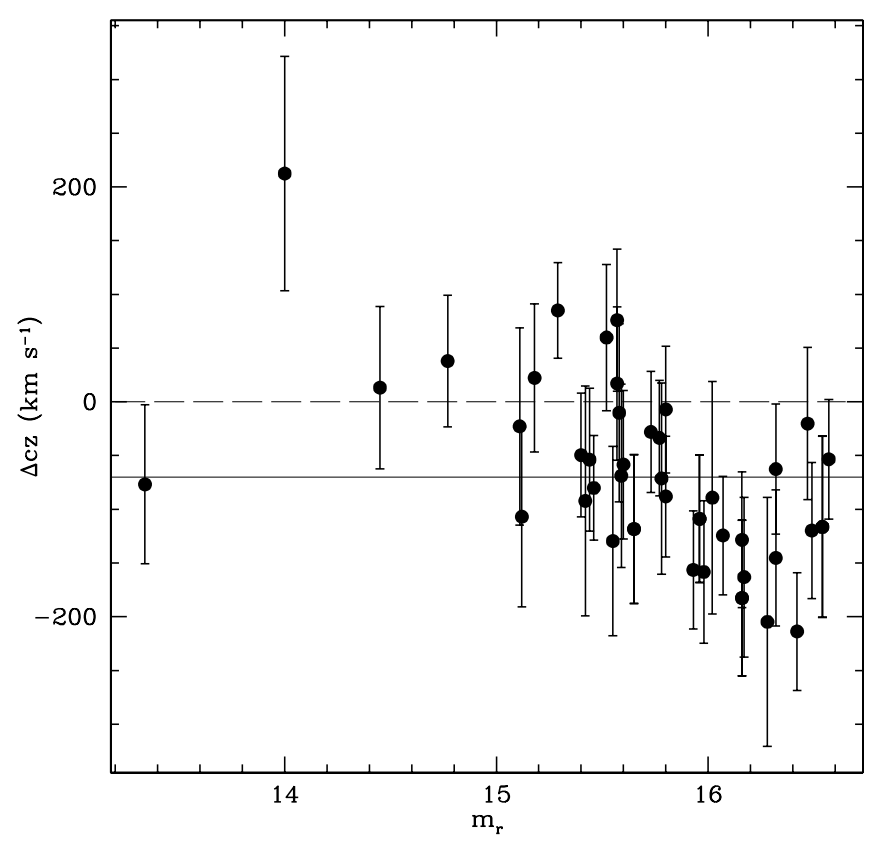

Fig. 3.-Difference between the velocities from Fabricant et al. (1989) and ours from the 1997 June and 1998 June runs. We find a mean difference of $70 \mathrm{~km} \mathrm{~s}^{-1}$ with a standard deviation of $84 \mathrm{~km} \mathrm{~s}^{-1}$. The error bars are $1 \sigma$ error bars as calculated from the quadratic sum of the two uncertainties.

Figure 4 shows the fraction of galaxies with radial velocity measurements versus $R_{\mathrm{KC}}$ magnitude for the combined data set. All magnitudes were converted to the $R_{\mathrm{KC}}$ magnitude system for comparison. Galaxies without magnitude information were not used in the determination of the fraction of galaxies with radial velocities.

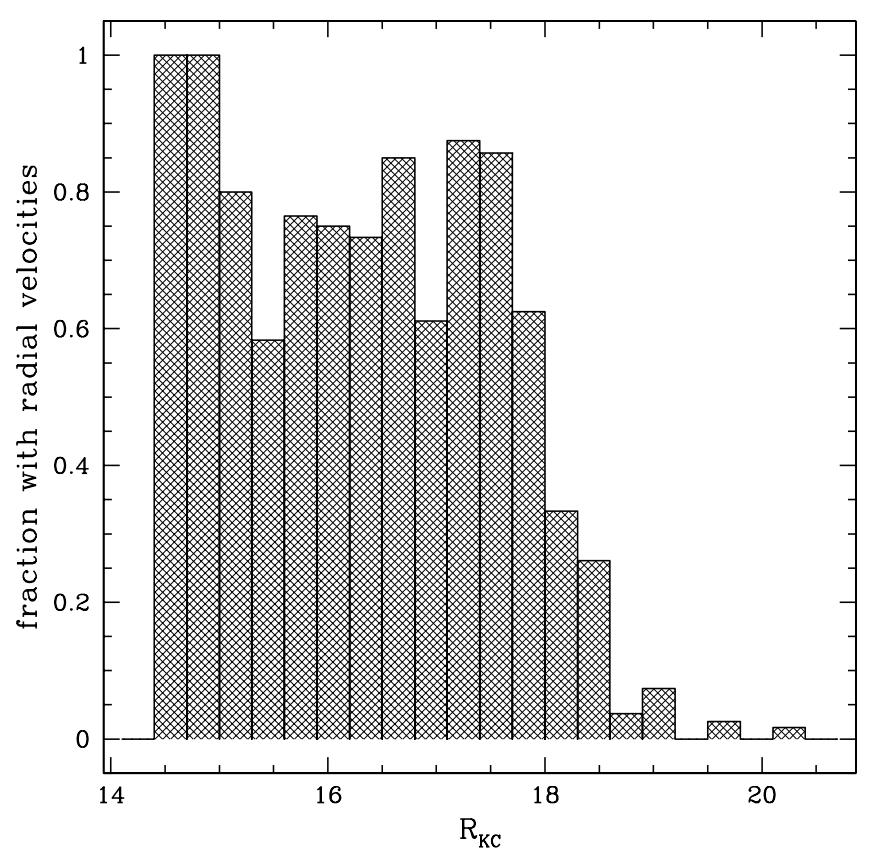

FIG. 4.-Fraction of galaxies with measured radial velocities as a function of $R_{\mathrm{KC}}$ magnitude. Only galaxies with measured magnitudes are included in the figure. All magnitudes from Fabricant et al. (1989) were converted to $R_{\mathrm{KC}}$ magnitude. As is shown above, the sample in Table 3 includes about $70 \%$ of the galaxies out to 18 th magnitude.

\subsection{Catalog}

We present the results of our galaxy spectroscopy in Table $3 .{ }^{3}$ Column (1) contains a galaxy identification number that will be used throughout this paper. Columns (2) and (3) give the equatorial coordinates of each galaxy in the J2000.0 epoch. All galaxies are sorted by increasing R.A. Column (4) contains the total $R_{\mathrm{KC}}$-band magnitude of the galaxy as reported by FOCAS. The previous studies of Faber \& Dressler (1977) and Fabricant et al. (1989) provide isophotal magnitudes. We first compared the Fabricant et al. (1989) $r$ magnitudes with our $R_{\mathrm{KC}}$ magnitudes and found a systematic difference that is similar to what they noted when comparing their magnitudes with the $F$ magnitudes reported by Faber \& Dressler (1977). Column (5) reports the $1 \sigma$ error on the $R_{\mathrm{KC}}$ magnitudes reported in column (4). Column (6) presents the radial velocities obtained either in the present study or in the previous studies of Faber \& Dressler (1977) and Fabricant et al. (1989). The new velocity measurement is reported for those galaxies that were remeasured. Column (7) reports the $1 \sigma$ velocity errors of the velocities in column (5). As indicated in column (5), these errors are either as reported from the IRAF FXCOR package or as reported in the previous studies. All cross identifications between Fabricant et al. (1989) and the current work are indicated in column (8).

\section{ANALYSIS}

\subsection{The Search for Substructure}

If a galaxy cluster is a dynamically relaxed system, then the spatial distribution of galaxies should be approximately spherical and the velocity distribution should be approximately Gaussian. The presence of substructure indicates a departure from this quasi-equilibrium state. Substructure is indicated by at least one of the following: (1) significant multiple peaks in the galaxy position distribution, (2) significant departures from a single-Gaussian velocity distribution, and (3) correlated deviations from the global velocity and position distributions. This section presents the results of a battery of statistical tests that we applied to search for such substructure. Table 4 provides a brief description of each test that we applied to the data, along with references to more thorough descriptions.

Following Yahil \& Vidal (1977), we performed a $3 \sigma$ clip on the velocity sample to filter out likely foreground and background galaxies. An alternative technique to filter out the foreground and background galaxies, based on gaps in the velocity histogram, has been proposed by Zabludoff et al. (1993). We chose the $3 \sigma$ clip technique because the sample returned from the alternative technique differed by at most two galaxies at the high-redshift end of the velocity histogram. All the statistical tests were run on both the $3 \sigma$ clip data set and a $5 \sigma$ clip data set. The results were unaffected by the different populations. The $3 \sigma$ clip reduced the sample of 319 galaxies to 277 members. The galaxies removed from the sample consisted largely of galaxies from

\footnotetext{
${ }^{3}$ Table 3 contains only the galaxies with measured redshifts. The entire galaxy catalog of 1090 galaxies that combines those galaxies obtained from Fabricant et al. (1989) and the WIYN $3 \times 3$ mosaic will be made available electronically from the Astronomical Data Center (ADC). The ADC's Internet site hosts World Wide Web and ftp access to the ADC's archives at http://www.gsfc.nasa.gov.
} 
TABLE 3

Galaxy Catalog for A2256

\begin{tabular}{|c|c|c|c|c|c|c|c|}
\hline $\begin{array}{c}\text { Number } \\
\text { (1) }\end{array}$ & $\begin{array}{l}\text { R.A. } \\
(\mathrm{J} 2000.0) \\
(2)\end{array}$ & $\begin{array}{c}\text { Decl. } \\
(\mathrm{J} 2000.0) \\
(3)\end{array}$ & $\begin{array}{l}R_{\mathrm{KC}} \\
\text { (4) }\end{array}$ & $\begin{array}{l}m_{r} \\
(5)\end{array}$ & $\begin{array}{c}c z \\
\left(\mathrm{~km} \mathrm{~s}^{-1}\right) \\
(6)\end{array}$ & $\begin{array}{c}1 \sigma \text { Velocity Error } \\
\left(\mathrm{km} \mathrm{s}^{-1}\right) \\
(7)\end{array}$ & $\begin{array}{c}\text { Fabricant } \\
\text { Number } \\
(8)\end{array}$ \\
\hline $10 \ldots \ldots \ldots \ldots$ & 165405.8 & 783742.8 & & 14.66 & 16710.4 & 36.4 & 11 \\
\hline $13 \ldots \ldots \ldots$ & 165426.2 & 783656.2 & & & 16649.1 & 47.9 & \\
\hline $15 \ldots \ldots \ldots \ldots$ & 165426.3 & 783341.9 & & 14.72 & 16197.4 & 44.7 & 14 \\
\hline $17 \ldots \ldots \ldots \ldots$ & 165445.5 & 783307.8 & & & 16032.4 & 47.3 & \\
\hline $19 \ldots \ldots \ldots$ & 165511.5 & 783016.6 & & & 59267.5 & 49.2 & \\
\hline $20 \ldots \ldots \ldots \ldots$ & 165532.0 & 783202.3 & & 16.16 & 16179.6 & 34.9 & 17 \\
\hline $22 \ldots \ldots \ldots \ldots$ & 165550.5 & 785100.1 & & & 18346.6 & 44.8 & \\
\hline $23 \ldots \ldots \ldots \ldots$ & 165559.8 & 784615.7 & & 16.05 & 18436.9 & 52.0 & 19 \\
\hline $24 \ldots \ldots \ldots \ldots$ & 165609.2 & 785636.2 & & 15.75 & 17851.4 & 46.3 & 20 \\
\hline $25 \ldots \ldots \ldots \ldots$ & 165615.5 & 785509.1 & & & 18308.9 & 47.7 & \\
\hline $26 \ldots \ldots \ldots . .$. & 165618.8 & 783207.2 & & & 18374.3 & 33.7 & \\
\hline $28 \ldots \ldots \ldots \ldots$ & 165640.9 & 783704.5 & & & 17867.8 & 33.4 & \\
\hline $29 \ldots \ldots \ldots . . . .$. & 165647.2 & 784658.5 & & 16.40 & 16947.3 & 46.3 & 23 \\
\hline $30 \ldots \ldots \ldots \ldots$ & 165648.2 & 783130.0 & & & 59465.4 & 58.1 & \\
\hline $31 \ldots \ldots \ldots \ldots$ & 165656.6 & 785857.5 & & & 57833.1 & 52.5 & \\
\hline $33 \ldots \ldots \ldots \ldots$ & 165701.6 & 783927.6 & & 16.58 & 18835.7 & 46.8 & 24 \\
\hline $34 \ldots \ldots \ldots \ldots$ & 165705.5 & 783248.1 & & 16.04 & 16494.7 & 31.0 & 25 \\
\hline $35 \ldots \ldots \ldots \ldots$ & 165714.8 & 782120.4 & & & 59241.4 & 59.3 & \\
\hline $36 \ldots \ldots \ldots \ldots$ & 165717.5 & 784705.1 & & & 18199.9 & 34.9 & \\
\hline $37 \ldots \ldots \ldots \ldots$ & 165742.2 & 783530.7 & & & 19386.7 & 40.9 & \\
\hline $38 \ldots \ldots \ldots \ldots$ & 165753.1 & 783724.3 & & & 17432.1 & 47.6 & \\
\hline $39 \ldots \ldots \ldots \ldots$ & 165756.1 & 783830.1 & & & 18085.7 & 57.6 & \\
\hline $40 \ldots \ldots \ldots \ldots$ & 165758.7 & 783614.9 & & 15.77 & 4253.1 & 44.6 & 27 \\
\hline $41 \ldots \ldots \ldots . .$. & 165801.2 & 783346.0 & & 15.40 & 19202.0 & 40.0 & 28 \\
\hline $42 \ldots \ldots \ldots \ldots$ & 165804.7 & 783938.2 & & & 17975.8 & 39.4 & \\
\hline $43 \ldots \ldots \ldots \ldots$ & 165809.3 & 784305.0 & & & 17143.6 & 46.1 & \\
\hline $45 \ldots \ldots \ldots \ldots$ & 165818.0 & 782933.8 & & 14.77 & 17881.9 & 46.5 & 29 \\
\hline $46 \ldots \ldots \ldots \ldots$ & 165820.8 & 784326.1 & & & 15909.3 & 51.6 & \\
\hline $47 \ldots \ldots \ldots \ldots$ & 165842.4 & 790719.3 & & & 53343.8 & 60.9 & \\
\hline $48 \ldots \ldots \ldots . . .$. & 165846.6 & 782522.0 & & 15.18 & 15635.2 & 49.2 & 30 \\
\hline $49 \ldots \ldots \ldots \ldots$ & 165851.1 & 785511.6 & & & 18086.7 & 47.6 & \\
\hline $50 \ldots \ldots \ldots \ldots$ & 165851.4 & 783059.5 & & 16.11 & 18504.0 & 44.0 & 31 \\
\hline $51 \ldots \ldots \ldots . .$. & 165853.0 & 784616.1 & & & 19347.7 & 66.1 & \\
\hline $52 \ldots \ldots \ldots \ldots$ & 165911.5 & 783713.1 & & & 16629.6 & 42.9 & \\
\hline $53 \ldots \ldots \ldots \ldots$ & 165912.6 & 783332.7 & & & 59325.7 & 68.0 & \\
\hline $54 \ldots \ldots \ldots \ldots$ & 165919.4 & 784822.6 & & & 15126.3 & 38.5 & \\
\hline $55 \ldots \ldots \ldots \ldots$ & 165925.6 & 790512.6 & & 16.22 & 18143.1 & 57.1 & 33 \\
\hline $56 \ldots \ldots \ldots \ldots$ & 165930.8 & 784325.1 & & & 16943.7 & 37.3 & \\
\hline $57 \ldots \ldots \ldots . .$. & 165937.2 & 782943.1 & & 16.17 & 15639.8 & 55.0 & 32 \\
\hline $58 \ldots \ldots \ldots \ldots$ & 165942.8 & 783708.6 & & & 53413.1 & 49.8 & \\
\hline $59 \ldots \ldots \ldots$ & 165948.0 & 784204.3 & & & 16595.6 & 48.9 & \\
\hline $60 \ldots \ldots \ldots \ldots$ & 165949.0 & 785819.8 & & & 17638.5 & 36.4 & \\
\hline $61 \ldots \ldots \ldots . . . .$. & 170009.7 & 785925.7 & & & 52218.3 & 108.0 & \\
\hline $62 \ldots \ldots \ldots \ldots$ & 170010.7 & 790015.8 & & 16.44 & 17414.7 & 69.7 & 37 \\
\hline $63 \ldots \ldots \ldots \ldots$ & 170011.2 & 790332.1 & & & 17667.5 & 34.2 & \\
\hline $64 \ldots \ldots \ldots \ldots$ & 170015.7 & 783424.5 & & 16.30 & 18355.0 & 62.0 & 34 \\
\hline $65 \ldots \ldots \ldots \ldots$ & 170019.5 & 784837.1 & & 16.13 & 15332.0 & 47.0 & 38 \\
\hline $66 \ldots \ldots \ldots \ldots$ & 170020.0 & 783355.7 & & 16.71 & 16372.4 & 55.4 & 35 \\
\hline $67 \ldots \ldots \ldots . . . .$. & 170021.3 & 784720.2 & & 16.12 & 18931.0 & 32.0 & 40 \\
\hline $68 \ldots \ldots \ldots \ldots$ & 170023.1 & 784206.2 & & 16.77 & 17637.9 & 29.4 & 39 \\
\hline $69 \ldots \ldots \ldots$ & 170025.4 & 783039.8 & & 16.32 & 17358.3 & 30.8 & 36 \\
\hline $70 \ldots \ldots \ldots$ & 170025.5 & 784926.6 & & & 15375.6 & 51.7 & \\
\hline $71 \ldots \ldots \ldots . . .$. & 170027.9 & 783057.9 & & & 18153.8 & 29.7 & \\
\hline $72 \ldots \ldots \ldots$ & 170029.2 & 783804.6 & & 16.40 & 17775.0 & 54.0 & 41 \\
\hline $73 \ldots \ldots \ldots \ldots$ & 170029.9 & 785610.9 & & 16.39 & 15237.0 & 47.0 & 43 \\
\hline $74 \ldots \ldots \ldots \ldots$ & 170031.7 & 784012.3 & & 15.77 & 17887.3 & 40.7 & 42 \\
\hline $79 \ldots \ldots \ldots$ & 170039.7 & 785700.1 & & & 18383.2 & 44.7 & \\
\hline $80 \ldots \ldots \ldots . .$. & 170040.2 & 784110.0 & $16.77 \pm 0.08$ & & 16284.2 & 33.8 & \\
\hline $86 \ldots \ldots \ldots . .$. & 170050.7 & 784949.3 & & 16.31 & 17357.0 & 43.0 & 45 \\
\hline $87 \ldots \ldots \ldots$ & 170051.3 & 784349.2 & $18.10 \pm 0.08$ & & 15916.8 & 42.3 & \\
\hline $88 \ldots \ldots \ldots \ldots$ & 170052.0 & 784121.9 & $15.39 \pm 0.07$ & 15.89 & 17325.0 & 39.0 & 44 \\
\hline $96 \ldots \ldots \ldots \ldots$ & 170056.9 & 783613.0 & $16.74 \pm 0.07$ & & 18281.5 & 32.4 & \\
\hline $99 \ldots \ldots \ldots . . . .$. & 170059.0 & 784500.1 & $15.29 \pm 0.08$ & 15.58 & 16136.7 & 52.8 & 46 \\
\hline
\end{tabular}


TABLE 3-Continued

\begin{tabular}{|c|c|c|c|c|c|c|c|}
\hline $\begin{array}{l}\text { Number } \\
\text { (1) }\end{array}$ & $\begin{array}{l}\text { R.A. } \\
(\text { J2000.0) } \\
(2)\end{array}$ & $\begin{array}{c}\text { Decl. } \\
(\mathrm{J} 2000.0) \\
(3)\end{array}$ & $\begin{array}{l}R_{\mathrm{KC}} \\
\text { (4) }\end{array}$ & $\begin{array}{l}m_{r} \\
(5)\end{array}$ & $\begin{array}{c}c z \\
\left(\mathrm{~km} \mathrm{~s}^{-1}\right) \\
(6)\end{array}$ & $\begin{array}{c}1 \sigma \text { Velocity Error } \\
\left(\mathrm{km} \mathrm{s}^{-1}\right) \\
(7)\end{array}$ & $\begin{array}{c}\text { Fabricant } \\
\text { Number } \\
(8)\end{array}$ \\
\hline $101 \ldots \ldots \ldots$ & 170059.7 & 790510.0 & & 16.58 & 18233.8 & 47.7 & 48 \\
\hline $102 \ldots \ldots \ldots$ & 170059.9 & 784531.8 & $17.31 \pm 0.08$ & & 17027.8 & 46.8 & \\
\hline $116 \ldots \ldots \ldots$ & 170110.5 & 784336.3 & $15.08 \pm 0.08$ & 15.29 & 16122.9 & 43.3 & 47 \\
\hline $119 \ldots \ldots \ldots$ & 170111.2 & 790531.0 & & 16.18 & 18538.2 & 31.4 & 50 \\
\hline $125 \ldots \ldots \ldots$ & 170113.8 & 784727.9 & & & 18590.7 & 83.6 & \\
\hline $138 \ldots \ldots \ldots$ & 170120.2 & 784519.8 & $18.02 \pm 0.08$ & & 16969.8 & 15.1 & \\
\hline $142 \ldots \ldots \ldots$ & 170123.1 & 784115.8 & $16.28 \pm 0.08$ & 15.73 & 16217.0 & 67.0 & 49 \\
\hline $152 \ldots \ldots \ldots$ & 170129.4 & 783608.0 & $15.77 \pm 0.07$ & 16.16 & 17963.5 & 42.3 & 52 \\
\hline $154 \ldots \ldots \ldots$ & 170130.4 & 783152.5 & $16.73 \pm 0.08$ & 16.69 & 17674.6 & 67.6 & 51 \\
\hline $155 \ldots \ldots \ldots$ & 170131.2 & 784010.9 & $19.38 \pm 0.07$ & & 19673.6 & 15.3 & \\
\hline $157 \ldots \ldots \ldots$ & 170133.8 & 784957.3 & & & 15668.7 & 43.3 & \\
\hline $158 \ldots \ldots \ldots$ & 170134.3 & 785045.9 & & & 19518.4 & 15.1 & \\
\hline $166 \ldots \ldots \ldots$ & 170138.2 & 784316.7 & $17.09 \pm 0.08$ & & 17065.0 & 83.2 & \\
\hline $178 \ldots \ldots \ldots$ & 170143.8 & 783653.6 & $17.08 \pm 0.07$ & & 18096.1 & 85.0 & \\
\hline $179 \ldots \ldots \ldots$ & 170144.4 & 784606.2 & $17.86 \pm 0.08$ & & 51759.5 & 93.7 & \\
\hline $183 \ldots \ldots \ldots$ & 170146.4 & 785332.0 & & & 14864.3 & 36.5 & \\
\hline $186 \ldots \ldots \ldots$ & 170147.5 & 785123.9 & & & 34075.9 & 44.8 & \\
\hline $187 \ldots \ldots \ldots$ & 170148.8 & 790216.8 & & 13.79 & 19302.7 & 59.6 & 54 \\
\hline $200 \ldots \ldots \ldots$ & 170158.1 & 784238.4 & $16.65 \pm 0.08$ & & 14834.6 & 40.5 & \\
\hline $205 \ldots \ldots \ldots$ & 170203.5 & 783251.4 & $17.87 \pm 0.08$ & & 17506.9 & 37.2 & \\
\hline $207 \ldots \ldots \ldots . . . .$. & 170205.0 & 784546.0 & $15.53 \pm 0.08$ & 15.78 & 17698.6 & 76.2 & 55 \\
\hline $208 \ldots \ldots \ldots$ & 170205.8 & 781729.1 & & 15.55 & 18001.0 & 47.0 & 53 \\
\hline $209 \ldots \ldots \ldots$ & 170206.3 & 782821.6 & & & 16249.2 & 82.3 & \\
\hline $215 \ldots \ldots \ldots$ & 170210.3 & 784529.6 & $15.78 \pm 0.08$ & 16.02 & 17988.0 & 43.0 & 57 \\
\hline $217 \ldots \ldots \ldots$ & 170210.9 & 785131.0 & & 16.12 & 19421.0 & 59.0 & 59 \\
\hline $225 \ldots \ldots \ldots$ & 170214.7 & 783547.7 & $15.87 \pm 0.08$ & 15.64 & 18756.9 & 39.3 & 56 \\
\hline $228 \ldots \ldots \ldots$ & 170216.3 & 785411.6 & & & 19559.3 & 48.2 & \\
\hline $230 \ldots \ldots \ldots$ & 170216.7 & 784317.4 & $16.05 \pm 0.08$ & 16.56 & 17991.6 & 37.7 & 60 \\
\hline $231 \ldots \ldots \ldots$ & 170216.8 & 783818.7 & $16.22 \pm 0.07$ & 16.67 & 15678.9 & 41.3 & 58 \\
\hline $232 \ldots \ldots \ldots$ & 170217.5 & 784552.6 & $16.29 \pm 0.08$ & 16.74 & 19907.4 & 66.3 & 61 \\
\hline $233 \ldots \ldots \ldots$ & 170218.1 & 784603.8 & $15.62 \pm 0.08$ & 15.96 & 19643.0 & 43.8 & 63 \\
\hline $236 \ldots \ldots \ldots$ & 170219.8 & 783613.1 & $16.08 \pm 0.07$ & & 15577.5 & 75.7 & \\
\hline $240 \ldots \ldots \ldots$ & 170221.3 & 784502.4 & $16.82 \pm 0.08$ & & 17550.1 & 33.5 & \\
\hline $242 \ldots \ldots \ldots$ & 170222.4 & 783538.5 & $16.16 \pm 0.08$ & 16.61 & 16705.0 & 34.2 & 62 \\
\hline $243 \ldots \ldots \ldots$ & 170223.3 & 790616.3 & & & 17300.0 & 46.0 & \\
\hline $249 \ldots \ldots \ldots$ & 170226.6 & 784449.9 & $16.56 \pm 0.08$ & & 16865.1 & 36.4 & \\
\hline $251 \ldots \ldots \ldots$ & 170227.5 & 785505.7 & & & 15036.8 & 79.8 & \\
\hline $256 \ldots \ldots \ldots$ & 170231.3 & 784305.4 & $16.19 \pm 0.08$ & 16.68 & 15419.0 & 60.0 & 64 \\
\hline $262 \ldots \ldots \ldots$ & 170234.2 & 783937.6 & $16.25 \pm 0.07$ & & 17800.3 & 30.7 & \\
\hline $265 \ldots \ldots \ldots$. & 170234.6 & 783128.9 & $17.56 \pm 0.08$ & & 16665.0 & 65.4 & \\
\hline $266 \ldots \ldots \ldots$ & 170235.7 & 784812.5 & & & 16828.9 & 36.6 & \\
\hline $267 \ldots \ldots \ldots$ & 170236.0 & 784558.3 & $16.04 \pm 0.08$ & & 18918.0 & 35.6 & \\
\hline $270 \ldots \ldots \ldots$ & 170237.0 & 784036.0 & $16.63 \pm 0.07$ & & 17779.4 & 37.0 & \\
\hline $272 \ldots \ldots \ldots$ & 170238.1 & 783334.3 & $16.05 \pm 0.08$ & 16.60 & 15453.2 & 44.8 & 65 \\
\hline $294 \ldots \ldots \ldots . . . . .$. & 170246.3 & 785146.7 & & 15.52 & 16573.7 & 52.6 & 69 \\
\hline $295 \ldots \ldots \ldots$ & 170246.8 & 782546.7 & & & 53624.7 & 114.0 & \\
\hline $297 \ldots \ldots \ldots$ & 170247.5 & 783717.7 & $16.85 \pm 0.07$ & & 62676.0 & 109.0 & \\
\hline $298 \ldots \ldots \ldots$ & 170247.6 & 784427.9 & $14.58 \pm 0.08$ & 14.45 & 20119.5 & 54.1 & 68 \\
\hline $301 \ldots \ldots \ldots$ & 170248.9 & 783829.6 & $15.86 \pm 0.07$ & 15.92 & 16936.0 & 39.0 & 67 \\
\hline $310 \ldots \ldots \ldots$ & 170255.2 & 783504.5 & $16.41 \pm 0.07$ & & 16356.5 & 109.0 & \\
\hline $320 \ldots \ldots \ldots$ & 170257.7 & 784206.3 & $17.40 \pm 0.08$ & & 15976.8 & 67.7 & \\
\hline $324 \ldots \ldots \ldots$ & 170258.3 & 783817.1 & $17.20 \pm 0.07$ & & 15763.1 & 34.4 & \\
\hline $325 \ldots \ldots \ldots$ & 170258.5 & 784947.7 & & & 17654.6 & 47.1 & \\
\hline $332 \ldots \ldots \ldots$. & 170301.1 & 783652.5 & $15.76 \pm 0.07$ & 16.16 & 16545.0 & 35.0 & 71 \\
\hline $335 \ldots \ldots \ldots$. & 170302.4 & 783556.1 & $15.06 \pm 0.07$ & 15.05 & 16303.0 & 100.0 & 72 \\
\hline $347 \ldots \ldots \ldots$ & 170308.8 & 784121.0 & $16.11 \pm 0.07$ & 16.74 & 14280.8 & 49.3 & 74 \\
\hline $351 \ldots \ldots \ldots$ & 170310.4 & 781440.9 & & 15.89 & 17973.4 & 48.3 & 70 \\
\hline $353 \ldots \ldots \ldots$ & 170311.3 & 784031.1 & $15.80 \pm 0.07$ & 16.31 & 15901.0 & 39.0 & 75 \\
\hline $358 \ldots \ldots \ldots$. & 170312.0 & 783309.3 & $16.01 \pm 0.05$ & 16.42 & 16504.2 & 38.4 & 73 \\
\hline $360 \ldots \ldots \ldots$ & 170312.2 & 784021.4 & $17.88 \pm 0.07$ & 17.80 & 16637.0 & 52.0 & 76 \\
\hline $364 \ldots \ldots \ldots$. & 170313.0 & 784556.4 & $16.24 \pm 0.08$ & 16.65 & 15660.0 & 39.0 & 77 \\
\hline $369 \ldots \ldots \ldots$ & 170316.9 & 782646.4 & & & 16202.6 & 33.4 & \\
\hline $383 \ldots \ldots \ldots$ & 170321.5 & 784354.1 & $16.48 \pm 0.08$ & & 15060.2 & 66.0 & \\
\hline $385 \ldots \ldots \ldots$ & 170322.1 & 784656.2 & $16.18 \pm 0.08$ & & 19723.0 & 44.7 & \\
\hline
\end{tabular}


TABLE 3-Continued

\begin{tabular}{|c|c|c|c|c|c|c|c|}
\hline $\begin{array}{c}\text { Number } \\
\text { (1) }\end{array}$ & $\begin{array}{l}\text { R.A. } \\
(\mathrm{J} 2000.0) \\
(2)\end{array}$ & $\begin{array}{c}\text { Decl. } \\
(\mathrm{J} 2000.0) \\
(3)\end{array}$ & $\begin{array}{c}R_{\mathrm{KC}} \\
(4)\end{array}$ & $\begin{array}{l}m_{r} \\
(5)\end{array}$ & $\begin{array}{c}c z \\
\left(\mathrm{~km} \mathrm{~s}^{-1}\right) \\
(6)\end{array}$ & $\begin{array}{c}1 \sigma \text { Velocity Error } \\
\left(\mathrm{km} \mathrm{s}^{-1}\right) \\
(7)\end{array}$ & $\begin{array}{c}\text { Fabricant } \\
\text { Number } \\
\text { (8) }\end{array}$ \\
\hline $394 \ldots \ldots \ldots$ & 170324.8 & 782039.1 & & & 18886.0 & 47.9 & \\
\hline $396 \ldots \ldots \ldots$ & 170325.3 & 783530.8 & $17.53 \pm 0.07$ & & 16018.4 & 94.2 & \\
\hline $399 \ldots \ldots \ldots$ & 170326.0 & 785242.7 & & & 19105.9 & 40.5 & \\
\hline $407 \ldots \ldots \ldots$ & 170328.3 & 783651.5 & $16.21 \pm 0.07$ & 16.71 & 17901.8 & 59.3 & 78 \\
\hline $410 \ldots \ldots \ldots$ & 170329.2 & 783755.7 & $15.02 \pm 0.07$ & 14.00 & 17796.4 & 43.5 & 79 \\
\hline $414 \ldots \ldots \ldots$ & 170329.7 & 783955.1 & $15.07 \pm 0.07$ & 15.30 & 17558.0 & 36.0 & 80 \\
\hline $417 \ldots \ldots \ldots$ & 170332.5 & 783702.9 & $17.58 \pm 0.07$ & & 16798.6 & 45.0 & \\
\hline $420 \ldots \ldots \ldots$ & 170333.8 & 783748.3 & $14.54 \pm 0.07$ & 14.20 & 16928.9 & 47.0 & 81 \\
\hline $422 \ldots \ldots \ldots$ & 170334.1 & 785055.2 & & 16.28 & 18943.2 & 49.2 & 85 \\
\hline $426 \ldots \ldots \ldots$ & 170335.3 & 783954.6 & $16.09 \pm 0.07$ & 16.57 & 15425.6 & 43.2 & 82 \\
\hline $428 \ldots \ldots \ldots$ & 170335.6 & 783745.1 & $14.34 \pm 0.07$ & 14.00 & 15830.0 & 100.0 & 83 \\
\hline $431 \ldots \ldots \ldots$ & 170336.0 & 784744.0 & & 14.84 & 19800.0 & 100.0 & 86 \\
\hline $436 \ldots \ldots \ldots$ & 170336.9 & 784031.1 & $17.26 \pm 0.07$ & & 16980.6 & 35.1 & \\
\hline $438 \ldots \ldots \ldots$ & 170337.5 & 783827.7 & $16.15 \pm 0.07$ & 16.63 & 17628.0 & 46.0 & 84 \\
\hline $439 \ldots \ldots \ldots$ & 170337.8 & 784658.6 & $17.23 \pm 0.08$ & & 19856.1 & 101.0 & \\
\hline $440 \ldots \ldots \ldots$ & 170337.9 & 783654.8 & $16.95 \pm 0.07$ & & 15848.1 & 74.2 & \\
\hline $444 \ldots \ldots \ldots$ & 170339.1 & 782218.7 & & & 53949.6 & 84.9 & \\
\hline $448 \ldots \ldots \ldots$ & 170341.9 & 783916.7 & $18.51 \pm 0.07$ & & 14742.2 & 44.3 & \\
\hline $456 \ldots \ldots \ldots$ & 170346.3 & 783724.4 & $17.39 \pm 0.07$ & & 17885.8 & 37.7 & \\
\hline $458 \ldots \ldots \ldots$ & 170346.8 & 783435.9 & $16.72 \pm 0.05$ & & 17323.9 & 40.2 & \\
\hline $470 \ldots \ldots \ldots$ & 170350.3 & 783702.9 & $16.30 \pm 0.07$ & 16.75 & 20245.0 & 41.9 & 88 \\
\hline $477 \ldots \ldots \ldots$ & 170353.3 & 782911.4 & $17.48 \pm 0.05$ & & 16265.6 & 52.9 & \\
\hline $484 \ldots \ldots \ldots$ & 170355.5 & 784420.5 & $17.51 \pm 0.08$ & & 15648.7 & 72.2 & \\
\hline $486 \ldots \ldots \ldots$ & 170356.2 & 784443.9 & $15.59 \pm 0.08$ & 15.80 & 17115.2 & 36.2 & 91 \\
\hline $487 \ldots \ldots \ldots$ & 170356.3 & 783736.3 & $15.76 \pm 0.07$ & 16.16 & 15151.1 & 34.5 & 89 \\
\hline $495 \ldots \ldots \ldots$ & 170357.6 & 784126.4 & $16.10 \pm 0.08$ & 16.50 & 20404.0 & 46.0 & 90 \\
\hline $498 \ldots \ldots \ldots$ & 170358.4 & 783234.7 & $16.67 \pm 0.05$ & & 17010.7 & 53.9 & \\
\hline $512 \ldots \ldots \ldots$ & 170403.8 & 783323.2 & $16.28 \pm 0.05$ & & 17355.3 & 53.1 & \\
\hline $516 \ldots \ldots \ldots$ & 170405.6 & 783150.7 & $17.34 \pm 0.05$ & & 16181.8 & 39.8 & \\
\hline $520 \ldots \ldots \ldots$ & 170408.0 & 782647.2 & & & 16803.9 & 53.1 & \\
\hline $526 \ldots \ldots \ldots$ & 170411.8 & 783449.0 & $16.87 \pm 0.05$ & & 19642.6 & 38.5 & \\
\hline $530 \ldots \ldots \ldots$ & 170413.1 & 783744.3 & $14.20 \pm 0.07$ & 13.92 & 16903.0 & 100.0 & 93 \\
\hline $538 \ldots \ldots \ldots$ & 170414.3 & 785435.1 & & & 18981.0 & 44.7 & \\
\hline $543 \ldots \ldots \ldots$ & 170416.1 & 784045.7 & $15.48 \pm 0.07$ & 15.99 & 15690.0 & 72.0 & 94 \\
\hline $547 \ldots \ldots \ldots$ & 170417.0 & 784732.3 & & & 19233.4 & 39.4 & \\
\hline $565 \ldots \ldots \ldots$ & 170420.9 & 783622.3 & $17.79 \pm 0.07$ & & 17409.3 & 71.9 & \\
\hline $568 \ldots \ldots \ldots$ & 170421.6 & 784230.5 & $16.42 \pm 0.08$ & & 14994.1 & 79.9 & \\
\hline $581 \ldots \ldots \ldots$ & 170426.8 & 783826.1 & $14.18 \pm 0.07$ & 13.34 & 17797.5 & 40.0 & 95 \\
\hline $585 \ldots \ldots \ldots$ & 170427.9 & 782519.1 & & & 16966.3 & 30.2 & \\
\hline $586 \ldots \ldots \ldots$ & 170428.1 & 784528.3 & $18.20 \pm 0.08$ & & 17533.3 & 135. & \\
\hline $591 \ldots \ldots \ldots$ & 170429.7 & 783848.0 & $17.04 \pm 0.07$ & & 16752.0 & 77.4 & \\
\hline $597 \ldots \ldots \ldots$ & 170432.6 & 784220.4 & $16.74 \pm 0.08$ & & 15188.8 & 38.3 & \\
\hline $605 \ldots \ldots \ldots$ & 170435.6 & 784005.9 & $17.34 \pm 0.07$ & & 19700.9 & 40.0 & \\
\hline $606 \ldots \ldots \ldots$ & 170435.9 & 782247.2 & & & 17492.8 & 34.3 & \\
\hline $607 \ldots \ldots \ldots$ & 170437.0 & 785321.1 & & & 18122.8 & 40.8 & \\
\hline $616 \ldots \ldots \ldots$ & 170440.2 & 784131.2 & $17.63 \pm 0.08$ & & 18596.9 & 128.0 & \\
\hline $617 \ldots \ldots \ldots$ & 170440.2 & 783742.0 & $16.61 \pm 0.07$ & & 19674.9 & 103.0 & \\
\hline $620 \ldots \ldots \ldots$ & 170440.6 & 781349.5 & & 16.41 & 18008.1 & 56.1 & 96 \\
\hline $621 \ldots \ldots \ldots$ & 170441.4 & 783209.1 & $16.75 \pm 0.05$ & & 16717.9 & 33.8 & \\
\hline $623 \ldots \ldots \ldots$ & 170443.4 & 783722.8 & $15.58 \pm 0.07$ & 16.07 & 15105.5 & 42.6 & 97 \\
\hline $633 \ldots \ldots \ldots$ & 170447.1 & 784031.5 & $17.01 \pm 0.07$ & & 16600.8 & 86.3 & \\
\hline $634 \ldots \ldots \ldots$ & 170447.1 & 783433.9 & $16.34 \pm 0.05$ & & 18816.7 & 42.0 & \\
\hline $635 \ldots \ldots \ldots$ & 170447.7 & 783830.0 & $14.76 \pm 0.07$ & 14.76 & 19094.2 & 49.1 & 98 \\
\hline $637 \ldots \ldots \ldots$ & 170448.3 & 784821.3 & & 15.44 & 19663.1 & 46.9 & 100 \\
\hline $641 \ldots \ldots \ldots$ & 170448.9 & 783743.3 & $17.44 \pm 0.07$ & & 17215.2 & 37.8 & \\
\hline $647 \ldots \ldots \ldots$ & 170451.5 & 784027.3 & $16.64 \pm 0.07$ & & 17734.3 & 63.9 & \\
\hline $651 \ldots \ldots \ldots$ & 170453.6 & 782954.3 & $16.98 \pm 0.05$ & & 52295.4 & 114.0 & \\
\hline $656 \ldots \ldots \ldots$ & 170455.3 & 785107.2 & & & 19980.1 & 44.8 & \\
\hline $658 \ldots \ldots \ldots$ & 170455.8 & 783404.2 & $17.75 \pm 0.05$ & & 18478.0 & 45.1 & \\
\hline $659 \ldots \ldots \ldots$ & 170456.3 & 784306.4 & $17.09 \pm 0.08$ & & 18682.0 & 44.9 & \\
\hline $662 \ldots \ldots \ldots$ & 170457.4 & 783813.5 & $17.25 \pm 0.07$ & & 19525.8 & 76.5 & \\
\hline $664 \ldots \ldots \ldots$ & 170457.7 & 785004.8 & & 16.27 & 19590.0 & 52.0 & 104 \\
\hline $665 \ldots \ldots \ldots$ & 170458.2 & 784204.2 & $16.61 \pm 0.08$ & & 17351.7 & 37.8 & \\
\hline $667 \ldots \ldots \ldots$ & 170458.7 & 782457.6 & & 16.49 & 26841.1 & 49.0 & 99 \\
\hline
\end{tabular}


TABLE 3-Continued

\begin{tabular}{|c|c|c|c|c|c|c|c|}
\hline $\begin{array}{l}\text { Number } \\
\text { (1) }\end{array}$ & $\begin{array}{l}\text { R.A. } \\
(\text { J2000.0) } \\
(2)\end{array}$ & $\begin{array}{c}\text { Decl. } \\
(\mathrm{J} 2000.0) \\
(3)\end{array}$ & $\begin{array}{l}R_{\mathrm{KC}} \\
\text { (4) }\end{array}$ & $\begin{array}{l}m_{r} \\
(5)\end{array}$ & $\begin{array}{c}c z \\
\left(\mathrm{~km} \mathrm{~s}^{-1}\right) \\
(6)\end{array}$ & $\begin{array}{c}1 \sigma \text { Velocity Error } \\
\left(\mathrm{km} \mathrm{s}^{-1}\right) \\
(7)\end{array}$ & $\begin{array}{c}\text { Fabricant } \\
\text { Number } \\
(8)\end{array}$ \\
\hline $668 \ldots \ldots \ldots . . .$. & 170458.8 & 783505.1 & $16.23 \pm 0.07$ & 16.77 & 17135.9 & 44.4 & 102 \\
\hline $678 \ldots \ldots \ldots$ & 170503.0 & 784003.2 & $16.01 \pm 0.07$ & 16.40 & 16762.0 & 49.0 & 105 \\
\hline $684 \ldots \ldots \ldots . .$. & 170506.3 & 782822.8 & & 15.65 & 19248.6 & 57.8 & 103 \\
\hline $693 \ldots \ldots \ldots$. & 170510.0 & 783136.0 & $15.86 \pm 0.05$ & 16.36 & 16535.7 & 34.8 & 106 \\
\hline 697. & 170511.7 & 784814.9 & & 16.72 & 19942.3 & 47.8 & 107 \\
\hline $702 \ldots \ldots \ldots$ & 170513.8 & 782919.5 & $16.88 \pm 0.05$ & & 16484.4 & 37.3 & \\
\hline $708 \ldots \ldots \ldots$ & 170515.2 & 782951.1 & $18.73 \pm 0.05$ & & 18146.7 & 39.8 & \\
\hline $709 \ldots \ldots \ldots$ & 170515.2 & 784812.8 & & & 42213.8 & 54.4 & \\
\hline $710 \ldots \ldots \ldots$ & 170515.5 & 783002.6 & $16.95 \pm 0.05$ & & 17939.0 & 73.7 & \\
\hline $712 \ldots \ldots \ldots$ & 170515.8 & 783237.5 & $16.90 \pm 0.05$ & & 18122.4 & 106.0 & \\
\hline $715 \ldots \ldots \ldots$ & 170518.1 & 783846.1 & $16.42 \pm 0.07$ & 16.55 & 17682.0 & 47.0 & 108 \\
\hline $725 \ldots \ldots \ldots$ & 170520.7 & 784720.9 & $17.85 \pm 0.08$ & & 17771.8 & 44.9 & \\
\hline $728 \ldots \ldots \ldots$ & 170521.1 & 790804.9 & & & 18806.5 & 48.3 & \\
\hline $731 \ldots \ldots \ldots$ & 170523.7 & 784023.8 & $17.38 \pm 0.07$ & & 18127.4 & 94.8 & \\
\hline $734 \ldots \ldots \ldots \ldots$ & 170525.0 & 782159.7 & & & 17321.5 & 36.2 & \\
\hline $738 \ldots \ldots \ldots$ & 170526.9 & 783847.8 & $16.45 \pm 0.07$ & 16.25 & 19599.0 & 57.0 & 109 \\
\hline $744 \ldots \ldots \ldots . .$. & 170530.3 & 784019.7 & $16.22 \pm 0.07$ & 16.20 & 17194.0 & 65.0 & 110 \\
\hline $745 \ldots \ldots \ldots$ & 170530.5 & 785515.3 & & & 17193.4 & 53.1 & \\
\hline $746 \ldots \ldots \ldots$ & 170531.5 & 783550.6 & $18.70 \pm 0.07$ & & 18652.4 & 44.2 & \\
\hline $747 \ldots \ldots \ldots$ & 170532.4 & 783902.1 & $16.83 \pm 0.07$ & & 15428.5 & 39.6 & \\
\hline $751 \ldots \ldots \ldots$ & 170533.1 & 784142.1 & $18.24 \pm 0.08$ & & 17789.6 & 41.1 & \\
\hline $752 \ldots \ldots \ldots$ & 170533.1 & 785117.1 & & 15.98 & 19478.6 & 53.7 & 113 \\
\hline $753 \ldots \ldots \ldots$ & 170533.5 & 783512.7 & $15.33 \pm 0.07$ & 15.27 & 18791.3 & 50.7 & 111 \\
\hline $755 \ldots \ldots \ldots$ & 170534.2 & 783034.2 & $17.56 \pm 0.05$ & & 19044.3 & 45.6 & \\
\hline $770 \ldots \ldots \ldots . .$. & 170539.1 & 783731.5 & $16.05 \pm 0.07$ & 15.59 & 16142.1 & 34.7 & 112 \\
\hline $780 \ldots \ldots \ldots . .$. & 170542.2 & 783625.4 & $17.39 \pm 0.07$ & & 17538.3 & 78.2 & \\
\hline $789 \ldots \ldots \ldots$ & 170546.9 & 782912.0 & $18.06 \pm 0.05$ & & 39704.7 & 48.0 & \\
\hline $792 \ldots \ldots \ldots$ & 170547.6 & 783957.5 & & & 18616.1 & 33.8 & \\
\hline $795 \ldots \ldots \ldots . .$. & 170548.6 & 782234.0 & & 15.94 & 16437.0 & 45.0 & 114 \\
\hline $798 \ldots \ldots \ldots$ & 170549.3 & 781618.4 & & & 51621.4 & 155.0 & \\
\hline $799 \ldots \ldots \ldots$ & 170549.5 & 783107.5 & $17.47 \pm 0.05$ & & 17701.6 & 135.0 & \\
\hline 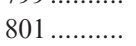 & 170550.2 & 782206.0 & & & 52074.8 & 65.0 & \\
\hline $822 \ldots \ldots \ldots . .$. & 170600.1 & 790643.2 & & 15.74 & 17927.3 & 47.4 & 117 \\
\hline $847 \ldots \ldots \ldots .$. & 170611.1 & 782438.2 & & & 18317.7 & 47.9 & \\
\hline $848 \ldots \ldots \ldots . .$. & 170611.4 & 783250.4 & $15.14 \pm 0.05$ & 15.42 & 18401.7 & 38.4 & 115 \\
\hline $853 \ldots \ldots \ldots$. & 170614.2 & 783358.1 & $17.77 \pm 0.05$ & & 17388.7 & 38.2 & \\
\hline $855 \ldots \ldots \ldots .$. & 170615.4 & 782653.4 & & & 32062.0 & 84.6 & \\
\hline $877 \ldots \ldots \ldots$ & 170622.5 & 783420.7 & $17.01 \pm 0.05$ & & 16658.2 & 107.0 & \\
\hline $882 \ldots \ldots \ldots$. & 170624.0 & 790237.3 & & & 17585.6 & 37.9 & \\
\hline $889 \ldots \ldots \ldots . .$. & 170628.2 & 784913.9 & & & 19093.1 & 44.6 & \\
\hline $895 \ldots \ldots \ldots . .$. & 170631.3 & 782518.0 & & 15.61 & 15626.0 & 35.0 & 118 \\
\hline $897 \ldots \ldots \ldots$ & 170631.8 & 784208.9 & $18.09 \pm 0.08$ & & 18858.9 & 27.8 & \\
\hline $898 \ldots \ldots \ldots . .$. & 170632.2 & 782500.7 & & & 17957.7 & 61.8 & \\
\hline 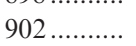 & 170634.9 & 782155.4 & & & 16799.8 & 43.3 & \\
\hline $903 \ldots \ldots \ldots . .$. & 170635.1 & 782600.2 & & & 27178.6 & 103.0 & \\
\hline $917 \ldots \ldots \ldots$ & 170639.7 & 782557.3 & & & 52150.3 & 92.5 & \\
\hline $921 \ldots \ldots \ldots$ & 170642.9 & 783558.3 & $16.27 \pm 0.07$ & 16.72 & 14909.8 & 77.2 & 120 \\
\hline $922 \ldots \ldots \ldots$ & 170643.0 & 783519.0 & $20.04 \pm 0.08$ & & 17954.2 & 154.0 & \\
\hline $934 \ldots \ldots \ldots .$. & 170648.6 & 783533.8 & & & 17408.7 & 76.3 & \\
\hline $935 \ldots \ldots \ldots .$. & 170649.5 & 782840.9 & $17.30 \pm 0.05$ & & 20079.3 & 56.8 & \\
\hline $939 \ldots \ldots \ldots . .$. & 170651.6 & 783342.5 & $16.42 \pm 0.05$ & & 18457.4 & 57.8 & \\
\hline $943 \ldots \ldots \ldots .$. & 170653.3 & 783056.1 & $15.63 \pm 0.05$ & 16.15 & 17998.0 & 61.0 & 121 \\
\hline $946 \ldots \ldots \ldots$ & 170656.0 & 784110.3 & $15.75 \pm 0.07$ & 15.60 & 16866.5 & 37.6 & 122 \\
\hline $948 \ldots \ldots \ldots$ & 170656.4 & 781514.4 & & & 16574.0 & 58.2 & \\
\hline $950 \ldots \ldots \ldots . . . . . .$. & 170656.6 & 782318.0 & & & 52117.8 & 99.6 & \\
\hline $956 \ldots \ldots \ldots$ & 170700.3 & 784124.3 & $17.38 \pm 0.07$ & & 17160.6 & 79.1 & \\
\hline $962 \ldots \ldots \ldots$ & 170701.7 & 791019.6 & & 16.43 & 11436.4 & 49.2 & 124 \\
\hline $969 \ldots \ldots \ldots$ & 170704.3 & 783557.7 & $17.49 \pm 0.07$ & & 15917.3 & 32.7 & \\
\hline $973 \ldots \ldots \ldots$ & 170707.4 & 783834.6 & $15.43 \pm 0.07$ & 15.80 & 18843.9 & 37.8 & 123 \\
\hline $980 \ldots \ldots \ldots . .$. & 170711.5 & 783858.4 & $17.15 \pm 0.07$ & & 17897.0 & 36.8 & \\
\hline $984 \ldots \ldots \ldots . .$. & 170713.4 & 785318.9 & & 15.93 & 16523.5 & 42.2 & 125 \\
\hline $987 \ldots \ldots \ldots . .$. & 170714.2 & 781912.4 & & & 17609.7 & 79.7 & \\
\hline $989 \ldots \ldots \ldots . .$. & 170715.5 & 783008.4 & $16.37 \pm 0.05$ & & 18581.5 & 47.5 & \\
\hline $999 \ldots \ldots \ldots$ & 170719.1 & 783950.5 & & & 18473.7 & 37.8 & \\
\hline
\end{tabular}


TABLE 3-Continued

\begin{tabular}{|c|c|c|c|c|c|c|c|}
\hline $\begin{array}{l}\text { Number } \\
\text { (1) }\end{array}$ & $\begin{array}{c}\text { R.A. } \\
(\mathrm{J} 2000.0) \\
(2)\end{array}$ & $\begin{array}{c}\text { Decl. } \\
(\mathrm{J} 2000.0) \\
(3)\end{array}$ & $\begin{array}{c}R_{\mathrm{KC}} \\
\text { (4) }\end{array}$ & $\begin{array}{l}m_{r} \\
(5)\end{array}$ & $\begin{array}{c}c z \\
\left(\mathrm{~km} \mathrm{~s}^{-1}\right) \\
(6)\end{array}$ & $\begin{array}{c}1 \sigma \text { Velocity Error } \\
\left(\mathrm{km} \mathrm{s}^{-1}\right) \\
(7)\end{array}$ & $\begin{array}{c}\text { Fabrican } \\
\text { Number } \\
\text { (8) }\end{array}$ \\
\hline $1002 \ldots \ldots \ldots$ & 170720.9 & 785337.4 & & & 16563.9 & 34.9 & \\
\hline $1004 \ldots \ldots .$. & 170720.9 & 782409.2 & & & 51293.1 & 108.0 & \\
\hline $1008 \ldots \ldots . .$. & 170726.6 & 783622.0 & & 16.47 & 17553.7 & 45.9 & 126 \\
\hline $1009 \ldots \ldots .$. & 170727.7 & 783640.2 & & 16.32 & 17637.6 & 47.2 & 127 \\
\hline $1010 \ldots \ldots \ldots$ & 170728.8 & 783008.4 & & & 19040.4 & 36.5 & \\
\hline $1011 \ldots \ldots$. & 170730.4 & 782441.8 & & & 51597.8 & 82.1 & \\
\hline $1012 \ldots \ldots \ldots$ & 170731.4 & 790836.8 & & 15.49 & 16603.7 & 38.6 & 128 \\
\hline $1013 \ldots \ldots$ & 170739.6 & 785656.0 & & & 53835.5 & 61.0 & \\
\hline $1014 \ldots \ldots .$. & 170754.9 & 784448.8 & & & 17027.4 & 38.2 & \\
\hline $1015 \ldots \ldots \ldots$ & 170758.5 & 781839.6 & & & 18428.3 & 42.0 & \\
\hline $1016 \ldots \ldots$ & 170802.0 & 781734.7 & & & 39920.8 & 78.6 & \\
\hline $1017 \ldots \ldots$. & 170802.9 & 783238.3 & & & 17910.5 & 34.2 & \\
\hline $1018 \ldots \ldots$. & 170805.4 & 783732.0 & & 16.29 & 17841.0 & 49.0 & 129 \\
\hline $1019 \ldots \ldots$ & 170805.7 & 782257.1 & & & 52032.8 & 81.9 & \\
\hline $1020 \ldots \ldots$. & 170809.2 & 783416.7 & & & 18541.1 & 47.1 & \\
\hline $1021 \ldots \ldots$. & 170810.6 & 783913.1 & & & 15020.8 & 72.5 & \\
\hline $1022 \ldots \ldots \ldots$ & 170813.9 & 784648.4 & & & 15957.6 & 53.6 & \\
\hline $1023 \ldots \ldots .$. & 170822.9 & 782228.1 & & & 17243.5 & 29.9 & \\
\hline $1024 \ldots \ldots \ldots$ & 170824.5 & 783428.8 & & & 18009.2 & 79.8 & \\
\hline $1025 \ldots \ldots \ldots$ & 170831.6 & 782137.6 & & 16.02 & 21638.7 & 52.3 & 130 \\
\hline $1026 \ldots \ldots$. & 170833.1 & 782954.4 & & 15.40 & 18684.4 & 46.3 & 131 \\
\hline $1027 \ldots \ldots$. & 170835.8 & 784031.6 & & & 17215.9 & 29.8 & \\
\hline $1028 \ldots \ldots .$. & 170841.3 & 782346.1 & & & 17017.6 & 65.2 & \\
\hline $1029 \ldots \ldots$. & 170845.6 & 783403.4 & & & 18347.9 & 34.3 & \\
\hline $1030 \ldots \ldots$. & 170846.3 & 783703.1 & & & 16512.8 & 37.3 & \\
\hline $1031 \ldots \ldots$. & 170847.5 & 782555.8 & & & 16860.2 & 6.85 & \\
\hline $1033 \ldots \ldots$. & 170857.7 & 785015.3 & & 15.57 & 16064.1 & 50.9 & 134 \\
\hline $1034 \ldots \ldots \ldots$ & 170858.7 & 783258.7 & & 16.75 & 16191.4 & 17.4 & 133 \\
\hline $1035 \ldots \ldots \ldots$ & 170859.0 & 783140.0 & & & 51442.5 & 112.0 & \\
\hline $1036 \ldots \ldots$. & 170900.6 & 784619.4 & & 15.46 & 11843.8 & 26.2 & 135 \\
\hline $1037 \ldots \ldots .$. & 170909.7 & 785232.0 & & 15.55 & 18811.8 & 46.3 & 137 \\
\hline $1039 \ldots \ldots \ldots$ & 170912.7 & 785056.8 & & & 52250.8 & 120.0 & \\
\hline $1040 \ldots \ldots$. & 170914.6 & 783810.8 & & & 18134.8 & 32.7 & \\
\hline $1041 \ldots \ldots$. & 170915.1 & 782158.4 & & & 16702.5 & 50.5 & \\
\hline $1042 \ldots \ldots \ldots$ & 170916.2 & 782711.6 & & & 7427.43 & 48.6 & \\
\hline $1043 \ldots \ldots$. & 170918.7 & 783016.8 & & & 16700.3 & 43.9 & \\
\hline $1044 \ldots \ldots .$. & 170922.4 & 782203.4 & & & 16193.1 & 63.5 & \\
\hline $1045 \ldots \ldots \ldots$ & 170923.9 & 784214.6 & & 16.39 & 17927.9 & 29.0 & 138 \\
\hline $1046 \ldots \ldots$. & 170930.2 & 784546.3 & & & 18249.8 & 44.8 & \\
\hline $1047 \ldots \ldots \ldots$ & 170939.5 & 783307.9 & & 15.73 & 16119.9 & 32.5 & 142 \\
\hline $1048 \ldots \ldots \ldots$ & 170943.2 & 783103.8 & & 16.63 & 18561.0 & 38.0 & 141 \\
\hline $1050 \ldots \ldots$. & 170944.9 & 782018.0 & & 15.12 & 17077.0 & 63.2 & 140 \\
\hline $1051 \ldots \ldots$. & 170945.8 & 783306.7 & & & 18694.1 & 58.3 & \\
\hline $1052 \ldots \ldots \ldots$ & 170946.6 & 784133.2 & & 16.58 & 17892.0 & 42.0 & 143 \\
\hline $1053 \ldots \ldots \ldots$ & 171007.0 & 783656.6 & & & 59089.3 & 51.8 & \\
\hline $1054 \ldots \ldots \ldots$ & 171013.4 & 783922.1 & & 16.44 & 17043.0 & 53.0 & 144 \\
\hline $1055 \ldots \ldots$. & 171027.4 & 782247.8 & & 16.70 & 17045.5 & 36.3 & 145 \\
\hline $1056 \ldots \ldots$. & 171031.2 & 782910.3 & & 16.54 & 15791.5 & 65.5 & 146 \\
\hline $1057 \ldots \ldots .$. & 171031.6 & 783351.3 & & 16.68 & 19464.0 & 34.1 & 147 \\
\hline $1058 \ldots \ldots \ldots$ & 171045.9 & 783215.3 & & 15.11 & 18767.0 & 31.0 & 148 \\
\hline $1059 \ldots \ldots$. & 171058.8 & 784526.1 & & & 27118.1 & 59.4 & \\
\hline $1060 \ldots \ldots$. & 171059.3 & 783652.4 & & & 41643.9 & 36.8 & \\
\hline $1061 \ldots \ldots$. & 171120.4 & 784947.7 & & & 39492.5 & 83.9 & \\
\hline $1062 \ldots \ldots$. & 171149.5 & 783552.7 & & & 17819.7 & 101.0 & \\
\hline $1065 \ldots \ldots \ldots$ & 171158.3 & 783626.8 & & & 42133.1 & 108.0 & \\
\hline $1068 \ldots \ldots \ldots$ & 171237.1 & 782830.9 & & 16.33 & 17125.5 & 67.1 & 153 \\
\hline $1069 \ldots \ldots$. & 171251.2 & 790339.6 & & 16.37 & 21886.4 & 45.4 & 156 \\
\hline $1071 \ldots \ldots$. & 171257.1 & 785644.1 & & & 19978.3 & 44.7 & \\
\hline $1072 \ldots \ldots \ldots$ & 171301.1 & 782902.8 & & 15.66 & 17540.5 & 50.0 & 155 \\
\hline $1073 \ldots \ldots$ & 171304.8 & 783424.2 & & & 19111.7 & 79.2 & \\
\hline $1074 \ldots \ldots .$. & 171308.5 & 783341.7 & & 15.90 & 17432.0 & 52.2 & 157 \\
\hline $1075 \ldots \ldots$. & 171308.6 & 783328.0 & & 14.95 & 15928.6 & 76.4 & 158 \\
\hline $1078 \ldots \ldots .$. & 171354.0 & 784629.9 & & & 25936.1 & 79.1 & \\
\hline $1080 \ldots \ldots$. & 171356.8 & 785933.5 & & 14.87 & 19613.7 & 61.5 & 164 \\
\hline
\end{tabular}


TABLE 4

Statistical Tests

\begin{tabular}{|c|c|c|}
\hline $\begin{array}{l}\text { Test } \\
(1)\end{array}$ & $\begin{array}{l}\text { Description } \\
\text { (2) }\end{array}$ & $\begin{array}{l}\text { References } \\
\text { (3) }\end{array}$ \\
\hline a..... & Average absolute deviation from mean velocity & $1,2,3$ \\
\hline ............ & Maximum velocity separation & 1,3 \\
\hline ........ & Variance of pairwise velocity separations & $1,3,4$ \\
\hline 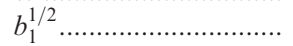 & Skewness of distribution & $1,5,6$ \\
\hline$b_{2} \ldots \ldots \ldots \ldots \ldots \ldots \ldots \ldots \ldots \ldots$ & Kurtosis of distribution & $1,3,5,6$ \\
\hline$b_{1}^{1 / 2} b_{2}$ Omnibus ............ & Skewness and kurtosis of distribution & 1,6 \\
\hline$I \ldots \ldots \ldots \ldots \ldots \ldots \ldots \ldots \ldots \ldots \ldots \ldots \ldots \ldots \ldots \ldots \ldots$ & $\begin{array}{l}\text { Ratio of standard deviation with median estimators } \\
\text { to standard deviation with biweight estimators }\end{array}$ & 1,7 \\
\hline$\ldots \ldots \ldots \ldots \ldots \ldots$ & Deviation of CDF ${ }^{\mathrm{a}}$ from best-fit Gaussian & 1,8 \\
\hline$\ldots \ldots \ldots \ldots \ldots \ldots \ldots$ & Deviation of CDF from best-fit Gaussian & 1,8 \\
\hline$W^{2}$ & Deviation of CDF from best-fit Gaussian & 1,8 \\
\hline$U^{2}$ & Deviation of CDF from best-fit Gaussian & 1,8 \\
\hline$A^{2} \ldots \ldots \ldots \ldots$ & Deviation of CDF from best-fit Gaussian & 1,8 \\
\hline Lee $2 \mathrm{D} \ldots \ldots \ldots \ldots \ldots \ldots \ldots \ldots$ & $\begin{array}{l}\text { Relative values of spaces and pairs in } \\
\text { two dimensions }\end{array}$ & $9,10,11$ \\
\hline Fourier elongation ....... & Elongation of spatial distribution & 11,12 \\
\hline$\Delta \ldots \ldots \ldots \ldots \ldots \ldots \ldots \ldots \ldots \ldots \ldots \ldots \ldots \ldots \ldots \ldots \ldots$ & Deviation of local mean and $\sigma$ from global values & 13 \\
\hline 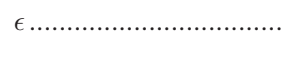 & $\begin{array}{l}\text { Change of projected mass estimator as a } \\
\text { function of positional space }\end{array}$ & 14,15 \\
\hline ....... & $\begin{array}{l}\text { Shift of spatial centroid as a function of } \\
\text { velocity space window }\end{array}$ & 11,16 \\
\hline Lee 3D ......................... & $\begin{array}{l}\text { Relative values of spaces and pairs in } \\
\text { three dimensions }\end{array}$ & $9,10,11$ \\
\hline
\end{tabular}

a Cumulative distribution function.

References.- (1) Beers et al. 1990. (2) Pearson \& Hartley 1962. (3) Yahil \& Vidal 1977. (4) Shapiro \& Wilk 1965. (5) Bird \& Beers 1993. (6) D’Agostino \& Stephens 1986. (7) Iglewicz 1983. (8) Stephens 1986. (9) Fitchett 1988. (10) Lee 1979. (11) Pinkney et al. 1996. (12) Rhee, van Haarlem, \& Katgert 1991. (13) Dressler \& Shectman 1988. (14) Bird 1994. (15) Heisler et al. 1985. (16)

West \& Bothun 1990.

a background group centered on the approximate sky coordinates $\alpha=17^{\mathrm{h}} 7^{\mathrm{m}}, \quad \delta=78^{\circ} 22^{\prime} \quad(\mathrm{J} 2000.0)$ and velocity $52,413.0 \mathrm{~km} \mathrm{~s}^{-1}$.

Each test listed in Table 4 requires a normalization procedure. For the one-dimensional tests, all calculations were normalized with respect to 10,000 bootstrapped intervals. The two-dimensional tests required an azimuthal shuffling of the data; 10,000 shuffles were used. For the three-dimensional tests, the velocities were randomized with respect to the positions. For all the three-dimensional tests, with the exception of the Lee-3D test, 10,000 randomizations were used. Because of the CPU cost, the Lee-3D test was applied with only 1000 shuffles. To calculate the confidence intervals of the tests, the value of the test statistic obtained from the original data was compared to the distribution of values from the shuffled data. The number of instances where the value of the test statistic was greater for the shuffled data than for the original data was divided by the total number of shufflings. This value is the confidence interval of the test. This bootstrap procedure is followed because the a priori probability distribution for the test statistic is often not known.

In Figure 5 we present the velocity histogram of the 277 galaxies that are likely cluster members, together with a single-Gaussian fit. While a quick visual inspection may suggest that the single Gaussian provides a reasonable representation of the distribution, a more detailed examination indicates several striking deviations from Gaussian behavior. The detailed statistical tests that we have applied support the conclusion that the structure is in fact nonGaussian. At high velocities, there is a striking group of gal- axies centered about $\sim 19,600 \mathrm{~km} \mathrm{~s}^{-1}$ and a relative deficit between 19,000 and 19,200 $\mathrm{km} \mathrm{s}^{-1}$. The remaining histogram has a strong peak, located at $17,900 \mathrm{~km} \mathrm{~s}^{-1}$, and between 15,000 and $19,000 \mathrm{~km} \mathrm{~s}^{-1}$ appears highly skewed to

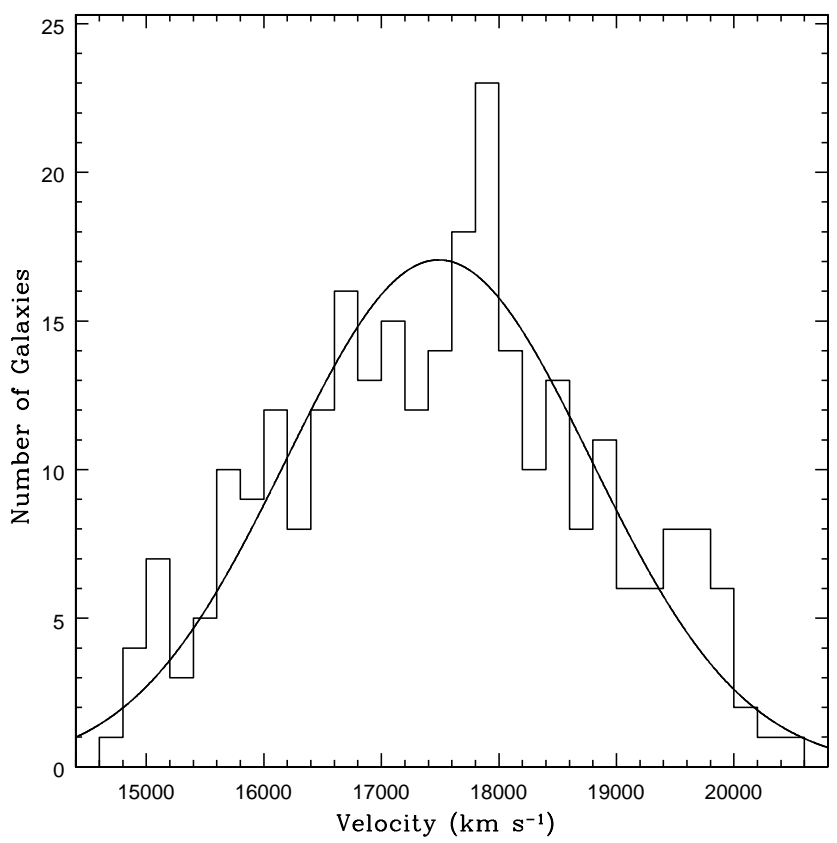

FIG. 5.-Velocity histogram for A2256. The solid line is the best singleGaussian fit to the data, with a mean of $17,488.1 \mathrm{~km} \mathrm{~s}^{-1}$ and a standard deviation of $1295.9 \mathrm{~km} \mathrm{~s}^{-1}$. 
TABLE 5

One-Dimensional Test Results

\begin{tabular}{|c|c|c|}
\hline $\begin{array}{c}\text { Statistical Test } \\
\text { (1) }\end{array}$ & $\begin{array}{l}\text { Value } \\
\text { (2) }\end{array}$ & $\begin{array}{l}\text { Significance } \\
\text { (3) }\end{array}$ \\
\hline a & 0.82 & 0.05 \\
\hline 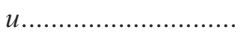 & 4.68 & $<0.05$ \\
\hline$W$ & 0.97 & $<0.01$ \\
\hline .................... & -0.011 & 0.47 \\
\hline$b_{2} \ldots \ldots \ldots \ldots \ldots \ldots \ldots \ldots \ldots \ldots \ldots \ldots \ldots$ & 2.36 & $<0.01$ \\
\hline$b_{1}^{1 / 2} b_{2}$ Omnibus ....... & 9.174 & 0.01 \\
\hline I & 0.94 & $>0.10$ \\
\hline KS & 0.50 & 0.25 \\
\hline ........ & 0.98 & 0.25 \\
\hline$W^{2} \ldots$ & 0.05 & 0.49 \\
\hline$U^{2} \ldots \ldots \ldots \ldots \ldots \ldots \ldots \ldots \ldots \ldots \ldots \ldots \ldots \ldots$ & 0.05 & 0.44 \\
\hline$A^{2} \ldots \ldots \ldots \ldots \ldots \ldots \ldots \ldots$ & 0.45 & 0.27 \\
\hline
\end{tabular}

the lower velocity end of the distribution at $17,900 \mathrm{~km} \mathrm{~s}^{-1}$. Furthermore, there is a strong correlation between velocity space and sky positional space that is unexpected from a relaxed system.

All one-dimensional tests performed are from the ROSTAT package (Beers, Flynn, \& Gebhardt 1990). The results are presented in Table 5. Column (1) gives the name of the statistic, column (2) gives the value of the statistic, and column (3) indicates the probability that this value can be obtained given the null hypothesis of no substructure. Low probability values provide significant evidence for substructure. In the case of the one-dimensional tests, the null hypothesis is that the velocity distribution is a single-component Gaussian. In Table 5, the $u$-test, the $W$-test, the $b_{2}$-test, the $b_{1}^{1 / 2} b_{2}$ Omnibus test, and marginally the $a$-test are the only ones that show significant deviations from a Gaussian in the $3 \sigma$ clipped data set.

A2256 has a nearly azimuthally symmetric galaxy distribution. This makes it difficult to detect substructure with two-dimensional tests, which are applied to the projected galaxy distribution. As a consequence, two-dimensional tests were not stressed in our search for substructure. The results of the two-dimensional tests are presented in Table 6 . In addition, it should be noted that tests that are sensitive to elongations or ellipticity, such as the Fourier elongation test, do not necessarily detect the presence of substructure. Two of the three two-dimensional tests showed evidence for substructure: the Lee-2D $l_{\max }$ and Lee-2D $l_{\text {rat }}$ tests. The results of the test indicate an elongation along a line that is oriented at a position angle of $120^{\circ}$. This direction is approximately the same as the apparent merging axis of the cluster.

With the large number of new velocities from our WIYNHydra spectra and the more accurate positions obtained from the WIYN images, it is natural to apply three-dimen-

TABLE 6

Two-Dimensional Test Results

\begin{tabular}{|c|c|c|}
\hline $\begin{array}{c}\text { Statistical Test } \\
\text { (1) }\end{array}$ & $\begin{array}{l}\text { Value } \\
\text { (2) }\end{array}$ & $\begin{array}{c}\text { Significance } \\
\text { (3) }\end{array}$ \\
\hline Lee $2 \mathrm{D} l_{\max } \cdot$ & 1.58 & 0.01 \\
\hline Lee $2 \mathrm{D} l_{\text {rat }} \ldots \ldots \ldots \ldots \ldots \ldots$ & 1.29 & $<0.01$ \\
\hline Fourier elongation ....... & 0.92 & 0.30 \\
\hline
\end{tabular}

TABLE 7

Three-Dimensional Test Results

\begin{tabular}{|c|c|c|}
\hline $\begin{array}{l}\text { Statistical Test } \\
\text { (1) }\end{array}$ & $\begin{array}{l}\text { Value } \\
\text { (2) }\end{array}$ & $\begin{array}{l}\text { Significance } \\
\text { (3) }\end{array}$ \\
\hline$\Delta \ldots \ldots \ldots \ldots \ldots \ldots \ldots$ & 374.1 & $<0.01$ \\
\hline$\epsilon \ldots \ldots \ldots \ldots \ldots \ldots \ldots$ & $2.24 \times 10^{15}$ & 0.68 \\
\hline$\alpha \ldots \ldots \ldots \ldots \ldots \ldots$ & 0.02 & 0.06 \\
\hline Lee $3 \mathrm{D} l_{\max } \ldots \ldots \ldots$ & 2.29 & 0.22 \\
\hline
\end{tabular}

sional tests to the data set. Of all the tests, the three-dimensional tests were the most CPU intensive, with the Lee-3D test at the top and the $\alpha$-test as the least computationally expensive test. Typical run times for the calculations ranged from 10 minutes for the $\alpha$-test on a $99 \mathrm{MHz} \mathrm{HP}-735$ workstation to $50 \mathrm{hr}$ for the Lee-3D test on a $233 \mathrm{MHz}$ Pentium II Linux system. The results of the tests are presented in Table 7.

A number of modifications have been made to the tests. Most importantly, the tests have been altered to use biweight estimators in place of classical estimators of the mean and standard deviations. In addition, the definition of a local sample has been changed to the $N_{\text {local }}=N^{1 / 2}$ nearest neighbors as suggested by Bird (1994), instead of the canonical 11 nearest neighbors. In our case $N_{\text {local }}=14$.

As can be seen in Table 7, two of the four three-dimensional tests, the Dressler-Shectman $\Delta$-test and $\alpha$-test, provide evidence for substructure at a $\gtrsim 94 \%$ confidence level. Figure 6 displays the Dressler-Shectman bubble plot for A2256. It is immediately apparent that there is a grouping of galaxies, to the north of the center, that deviate significantly from the global values. The galaxies that correspond to large circles in the bubble plot comprise the small clump of galaxies in the velocity histogram (Fig. 5) near 19,900 km

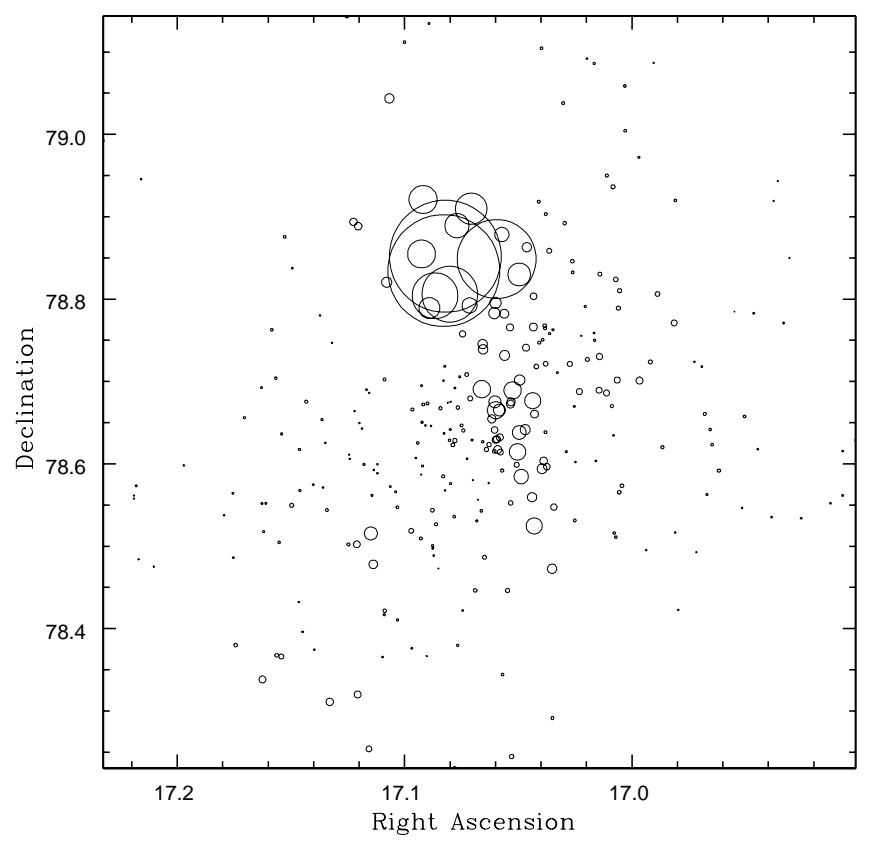

FIG. 6.-Dressler-Shectman plot for A2256. Each open circle is a galaxy whose radius is proportional to $e^{\delta} ; \delta$ is described by Dressler and Shectman as the deviation of the local mean and $\sigma$ from the global mean and $\sigma$. See text for an explanation. 
$\mathrm{s}^{-1}$. The Lee-3D test $l_{\max }$ did not show evidence for substructure. Since this test is designed for a bimodal distribution, it may be affected by the presence of a multimodal distribution.

The tests that show evidence for substructure are the $u$ test, the $b_{2}$-test, the $b_{1}^{1 / 2} b_{2}$ Omnibus test, the $W$-test, the Lee2D $l_{\max }$ and $l_{\text {rat }}$ tests, the Dressler-Shectman $\Delta$-test, and marginally the $a$-test. Each test is sensitive to a different departure from the null hypothesis of a unimodal Gaussian velocity distribution that is uncorrelated with an azimuthally symmetric galaxy distribution. Thus, it is not expected that all tests will reject this null hypothesis. The fact that six tests do reject the null hypothesis at a confidence of $\gtrsim 95 \%$ provides strong evidence for the presence of substructure.

\subsection{Mixture Modeling}

As indicated in $\S 3.1$, there is strong evidence for the presence of substructure in A2256. To quantify the nature of substructure, we employed the KMM software package (McLachlan \& Basford 1988), which was previously used for this application by Ashman, Bird, \& Zepf (1994). The KMM software fits a user-specified number of Gaussians to a multivariate distribution and provides a comparison of the quality of the multi-Gaussian fit relative to a singleGaussian fit. This software is based on the expectation maximization (EM) algorithm, which is described by McLachlan \& Krishnan (1997). Each element in the distribution is then assigned to one of the Gaussians based on maximum likelihood.

Figure 5 shows the velocity distribution histogram, along with the best single-Gaussian fit. At first glance, the singleGaussian model may appear to provide a reasonable fit, but the deviations are suspiciously large. To test the singleGaussian fit, we applied the Double Root Residual (DRR) test described by Gebhardt \& Beers (1991). The DRR test showed that the peak located at $18,000 \mathrm{~km} \mathrm{~s}^{-1}$ and the peak near $19,600 \mathrm{~km} \mathrm{~s}^{-1}$ are indeed significant deviations from the single-Gaussian fit.

To determine which multi-Gaussian model works best, we applied the KMM software to the velocity and position data set for models with two, three, and four Gaussian components. Thus, we have adopted a Gaussian model for the spatial distribution within each subclump as well as for the velocity distribution. The Gaussian model provides a convenient tool for deconvolving multiple spatial components, rather than an accurate model for the spatial structure of each subclump.

The triple-Gaussian model provided the best fit, and the result is shown in Figure 7. The mean, dispersion, and number of galaxies assigned to each of the Gaussians are given in Table 8. The improved fit of the triple-Gaussian model,

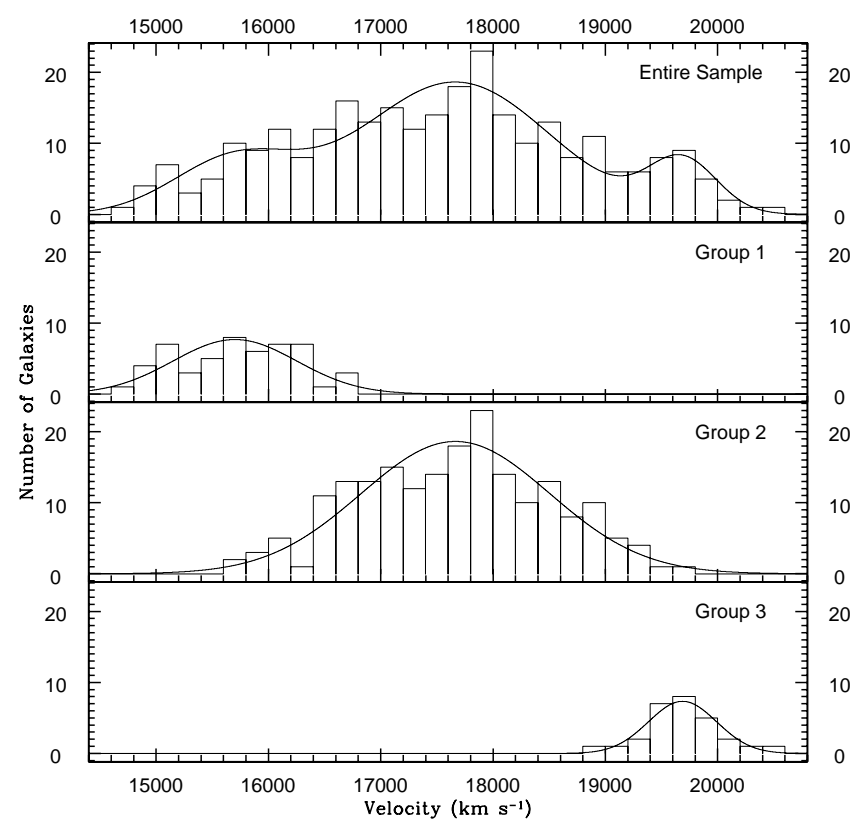

FIG. 7.-Velocity histogram for A2256 with the multiple Gaussian profiles. The top panel shows the combined profile from the multiple Gaussian model. The second, third, and bottom panels show the galaxies belonging to the appropriate groups 1,2, and 3, respectively, along with the best-fit Gaussian. Parameters for each Gaussian are described in Table 8.

relative to a single-Gaussian model, is significant at the $99 \%$ level. In Figure 7, it can be seen that Gaussian 3 corresponds well to the high-velocity clump centered at $\sim 19,600 \mathrm{~km} \mathrm{~s}^{-1}$.

We also investigated whether the large velocity clump marked by Gaussians 1 and 2 has significant substructure. To test for this, we removed the galaxies associated with Gaussian 3 and ran the same set of statistical tests on the 1-2 clump data set. All one-dimensional tests but the $I$ test showed significant deviations from a Gaussian distribution.

It is useful to observe the projected spatial distribution of the galaxies belonging to each subclump. This is shown in Figure 8 , where the symbol type plotted for each galaxy indicates its subclump membership. The localization of group 3 is immediately obvious. In contrast, the 1 and 2 groups have a large overlap. We interpret this as indicating that the 3 subclump is a small group in the process of merging with the $1-2$ system.

\section{DISCUSSION}

\subsection{Cluster Dynamics}

In $\S 3.2$ we presented evidence for the presence of three subclumps. Previous analyses, based on a smaller velocity sample, have detected only the 1 and 2 subclumps (Fabri-

TABLE 8

KMM Results

\begin{tabular}{|c|c|c|c|c|c|c|}
\hline $\begin{array}{l}\text { Gaussian } \\
\text { (1) }\end{array}$ & $\begin{array}{c}\text { R.A. } \\
(\mathrm{J} 2000.0) \\
(2)\end{array}$ & $\begin{array}{c}\text { Decl. } \\
(\mathrm{J} 2000.0) \\
(3)\end{array}$ & $\begin{array}{c}\text { Velocity Mean } \\
\left.\qquad \mathrm{km} \mathrm{s}^{-1}\right) \\
(4)\end{array}$ & $\begin{array}{c}\text { Velocity } \sigma \\
\left(\mathrm{km} \mathrm{s}^{-1}\right) \\
(5)\end{array}$ & $\begin{array}{l}N_{\text {gal }} \\
\text { (6) }\end{array}$ & $\begin{array}{c}M_{\mathrm{PME}} \\
\left(10^{15} M_{\odot}\right) \\
(7)\end{array}$ \\
\hline $1 \ldots \ldots \ldots \ldots \ldots$ & 170157 & 783937 & 15701.78 & 550.77 & 53 & 0.51 \\
\hline $2 \ldots \ldots \ldots \ldots$ & 170428 & 783835 & 17665.59 & 839.41 & 196 & 1.6 \\
\hline 3.................... & 170352 & 784608 & 19687.67 & 304.38 & 28 & 0.17 \\
\hline
\end{tabular}




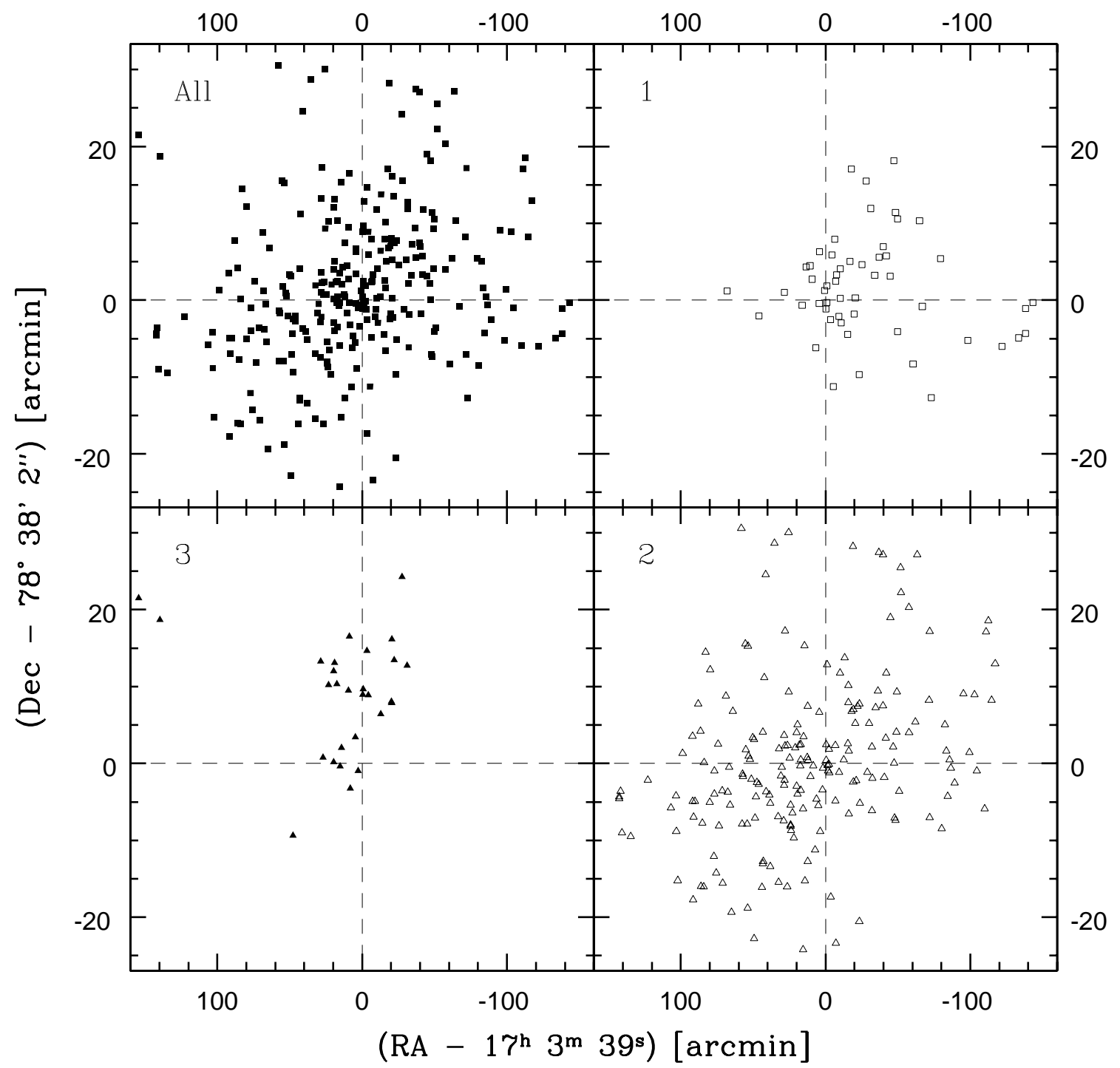

FIG. 8. - Sky plot of the galaxies associated with the three groups found by the KMM algorithm. The open squares represent galaxies that belong to group 1. The open triangles represent galaxies that belong to group 2 . The filled triangles are galaxies belonging to group 3 . The $x$-and $y$-axes give the offsets in arcminutes from the central brightest galaxy (ID 581).

cant et al. 1989; Briel et al. 1991; Roëttiger, Burns, \& Loken 1993). To check to see which systems are gravitationally bound, we applied the projected mass estimator as described by Heisler, Tremaine, \& Bahcall (1985). The masses are less than half the virial masses of the corresponding subclumps, potentially indicating that the system is not in virial equilibrium. In Table 8 the mass for each of the subclumps is given.

To perform the gravitational bound check, we can rewrite the Newtonian energy criterion for the two-body problem as

$$
V_{r}^{2} R_{p} \leq 2 G M \sin ^{2} \alpha \cos \alpha,
$$

where $V_{r}$ is the line-of-sight velocity, $R_{p}$ is the projected separation of the clump centers, $\alpha$ is the projection angle measured from the sky plane, and $M$ is the total mass of the system (Beers, Geller, \& Huchra 1982). Since equation (1) is based on a two-body analysis, we applied it to subclumps 1 and 3 in the following manner. For clump 1, we assumed that the mass of clump 3 is negligible, and the two point masses are subclumps 1 and 2. For group 3, we assumed that subclumps 1 and 2 are bound and constitute the other point mass. If all these systems are bound, they are likely to be interacting with each other. As a consequence, the firstranked galaxy (FRG) may not be located at the center of a local potential well. To minimize the effect of any FRG offsets, the position and velocity centroids of the galaxy groups defined in Table 8 are used to calculate the bound/unbound criteria. As will be shown in $\S 4.3$, the group centroids correspond well with the peaks seen in the X-ray data.

From Figures 9 and 10 a bound condition for groups 1 and 2 is satisfied between the projection angles of $20^{\circ}$ and $84^{\circ}$. Similarly, group 3 is bound for projection angles between $24^{\circ}$ and $81^{\circ}$.

\subsection{Dominant Galaxies}

Computer simulations by Bode et al. (1994) indicate that an FRG settles into the center of a poor cluster of galaxies 


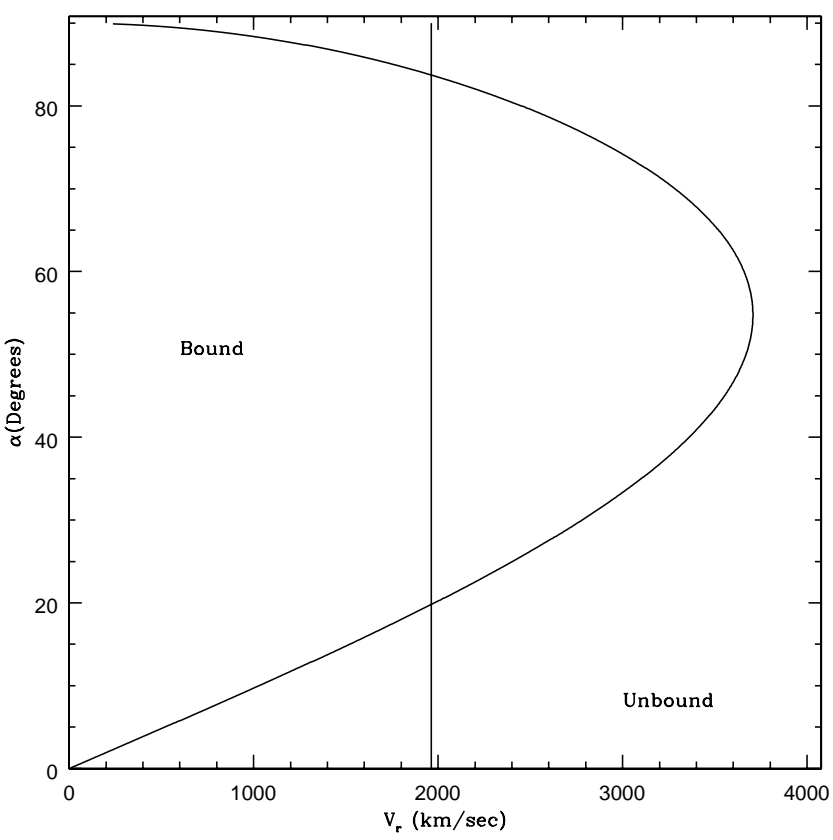

FIG. 9.-Plot showing the boundary between the bound and unbound orbits for groups 1 and 2. $V_{r}$ is the difference in the radial velocities between the two subclumps (see text). The angle between the sky plane and the line joining the centers of clusters 1 and 2 is $\alpha$. The vertical line marks the relative velocity difference between the two groups.

in a time less than the Hubble time. We shall define the FRG as the brightest galaxy within a defined group. The simulations show that the time that it takes this FRG to settle into the center is dependent on the velocity dispersion of the galaxies within the cluster. As the velocity dispersion increases, the rate of successful mergers decreases. Bode et al. (1994) argue that their models support the hypothesis

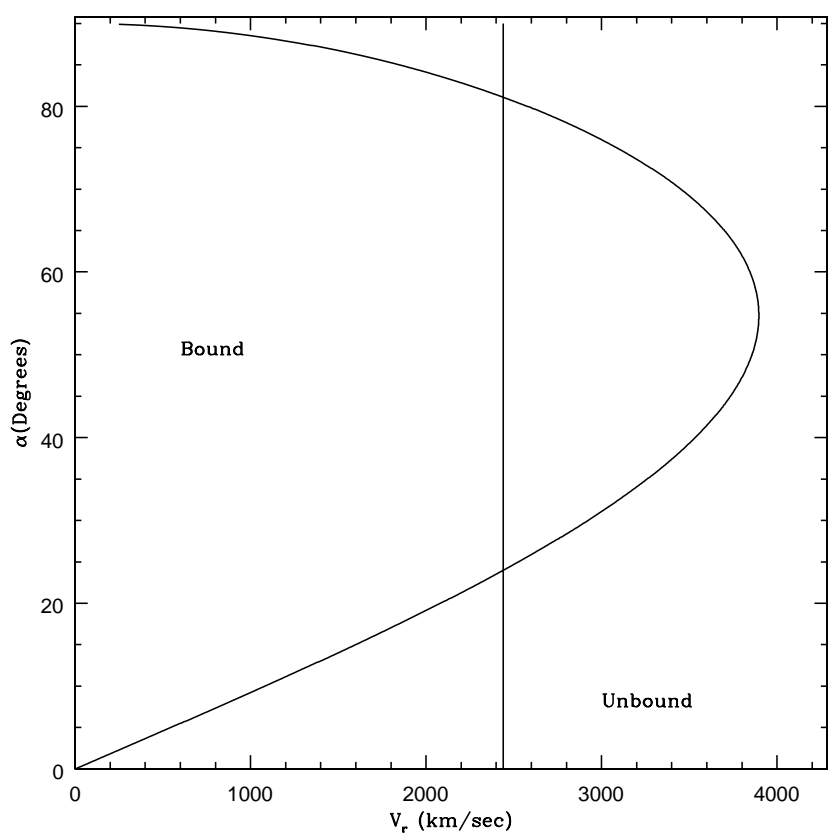

FIG. 10.- Same as Fig. 9, except that the two groups are the combination of groups 1 and 2 (group A) and group 3 (group B).

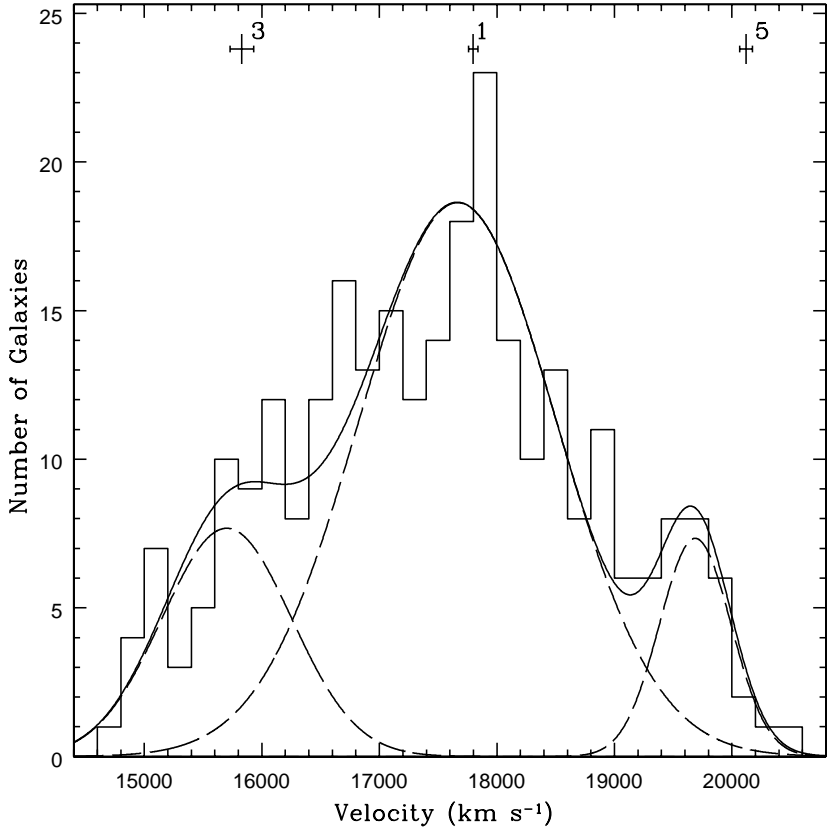

FIG. 11.-Histogram of the galaxies that are a member of A2256 with the triple-Gaussian model determined by application of the EM algorithm. The solid line shows the summed distribution of the combined system. The dashed line shows the individual Gaussians given in Table 8 . The velocity locations of the first, third, and fifth brightest galaxies, from Table 9, are indicated at the top of the diagram with $1 \sigma$ error bars.

that the FRG first forms in the central region of a poor cluster. In this environment, velocity dispersions are low and successful mergers are frequent, allowing the efficient development of a central dominant galaxy.

In Table 9 are listed the five brightest galaxies associated with A2256. The four brightest galaxies are found within the central region of the cluster. The fifth brightest galaxy is found centrally located within subclump 3 . Figure 11 shows the velocity histogram with the first, third, and fifth brightest galaxies marked to indicate their position within the three subclumps. The respective galaxies are closely associated with the corresponding groups not only in positional space but also in velocity space. The first and third galaxies are centrally located within the velocity distributions of subclumps 1 and 2, respectively. The fifth brightest galaxy is at the center of the velocity histogram feature associated with clump 3.

The second (ID 530) and fourth (ID 420) brightest galaxies are located within the central region of the cluster. Both galaxies are assigned to group 2 and have very similar velocities (see Table 9). These galaxies occupy an intermediate position between groups 1 and 2 in velocity space. One can speculate as to the true origin of these galaxies. Our merger hypothesis suggests that they are either FRGs from other poor groups that merged at some time in the past or second-ranked galaxies associated with groups 1 and 2 that have not yet merged with the central dominant galaxy of their respective group. Once these galaxies enter the environment of the merged system, the velocity dispersion increases. The chance of a merger occurring between the central dominant galaxies decreases significantly, and the second and fourth brightest galaxies may not merge with the first and third brightest galaxies for some time. We do 
TABLE 9

Brightest Galaxies IN A2256

\begin{tabular}{|c|c|c|c|c|c|c|c|}
\hline $\begin{array}{l}\text { Brightness Rank } \\
\text { (1) }\end{array}$ & $\begin{array}{l}\text { ID } \\
(2)\end{array}$ & $\begin{array}{c}\text { Fabricant ID } \\
\text { (3) }\end{array}$ & $\begin{array}{c}\text { R.A. } \\
\text { (J2000.0) } \\
(4)\end{array}$ & $\begin{array}{c}\text { Decl. } \\
(\mathrm{J} 2000.0) \\
(5)\end{array}$ & $\begin{array}{l}\text { Group } \\
(6)\end{array}$ & $\begin{array}{c}M_{R_{\mathrm{KC}}} \\
\quad(7)\end{array}$ & $\begin{array}{c}V_{r} \\
\left(\mathrm{~km} \mathrm{~s}^{-1}\right) \\
(8)\end{array}$ \\
\hline 1 & 581 & 95 & 170426.9 & 783826.1 & 2 & $14.19 \pm 0.07$ & $17797.6 \pm 40.0$ \\
\hline $2 \ldots \ldots \ldots \ldots \ldots \ldots \ldots \ldots \ldots \ldots \ldots \ldots \ldots \ldots$ & 530 & 93 & 170413.2 & 783744.3 & 2 & $14.20 \pm 0.07$ & $16903.0 \pm 100.0$ \\
\hline 3....................... & 428 & 83 & 170335.6 & 783745.1 & 1 & $14.35 \pm 0.07$ & $15830.0 \pm 100.0$ \\
\hline $4 \ldots \ldots \ldots \ldots \ldots \ldots \ldots \ldots$ & 420 & 81 & 170333.8 & 783748.3 & 2 & $14.55 \pm 0.07$ & $16929.0 \pm 47.1$ \\
\hline $5 \ldots \ldots \ldots \ldots \ldots \ldots \ldots \ldots \ldots \ldots \ldots \ldots \ldots \ldots$ & 298 & 68 & 170247.6 & 784427.9 & 3 & $14.59 \pm 0.08$ & $20119.5 \pm 54.2$ \\
\hline
\end{tabular}

not see evidence of groups corresponding to the second and fourth brightest galaxies, so we favor the second interpretation.

\subsection{X-Ray Data}

Several X-ray maps of A2256 are available in the literature (e.g., Briel et al. 1991; Briel \& Henry 1994; Markevitch 1996). It is one of the best examples of a cluster showing $\mathrm{X}$-ray substructure. The X-ray image shows two central peaks, separated by about 3.5 , that are located near the optical center of the cluster (Briel et al. 1991). As has been widely discussed, the X-ray emission can be used to trace the total gravitational potential of the cluster, under the assumption that the X-ray-emitting gas is in hydrostatic equilibrium.

If the luminous matter of the galaxies also traces the gravitational potential, then the substructures found by application of the EM algorithm should approximately correspond to the structure seen in the X-ray surface brightness distribution. The positions of the centers of groups 1 and 2 , listed in Table 8, correspond well with the two maxima observed in the extended cluster emission. Briel et al. (1991) mark the center of the central peak approximately $70^{\prime \prime}$ to the west of the central cD galaxy (ID 581). The center of group 2 is also approximately $70^{\prime \prime}$ to the northeast. This places the center of group 2 approximately $10^{\prime \prime}$ to the northeast of the central $\mathrm{cD}$ galaxy. The proximity of these positions strongly suggests that group 2 is the dominant group.

The position of group 1 is coincident with the position of the excess source defined by Briel et al. (1991). According to Table 9, the FRG for group 1 (ID 428) is located at $\alpha=17^{\mathrm{h}} 3^{\mathrm{m}} 35^{\mathrm{s}} \cdot 6, \delta=78^{\circ} 37^{\prime} 45^{\prime \prime} .1$, placing it only about 2.3 southeast of the excess source peak. The center of group 1 as obtained from the KMM algorithm is only approximately $5^{\prime}$ to the west of this peak. This also suggests a correlation between the excess source and the smaller infalling group 1 .

The left panels in Figure 12 show the position of each group along with the X-ray contours (Briel \& Henry 1994). The central dominant galaxy that corresponds to each group is marked by a star, and the centroids for groups 1,2, and 3 are marked by a cross. However, the small number of galaxies associated with group 3 makes the position for the centroid for group 3 uncertain. One should note that the centroid for group 3 is only 3.5 to the northeast of the FRG for group 3 (ID 298).

There are no other features in the X-ray surface brightness distribution that indicate the presence of a third group. This is not unexpected, since group 3 has a mass comparable to that of a poor group. Thus, the X-ray luminosity of group 3 is probably considerably less than those of groups 1 and 2 , and so the X-ray emission of A2256 is dominated by these brighter groups. Nevertheless, in $\S 4.4$ we will see that there is potential evidence in the radio emission for group 3.

\subsection{Radio Data}

In the right panels of Figure 12, we present radio contours (Röttgering et al. 1994) of the central region of A2256, along with the positions of the galaxies with measured redshifts. We shall use the same naming convention for features in the radio map as was used by Röttgering et al. (1994) and Bridle et al. (1979). For convenience, Table 10 includes a subset of the table provided by Röttgering et al. (1994). Column (1)

TABLE 10

Radio Source Positions in A2256

\begin{tabular}{|c|c|c|c|c|c|}
\hline $\begin{array}{l}\text { Component } \\
\text { (1) }\end{array}$ & $\begin{array}{c}\text { R.A. } \\
\text { (J2000.0) } \\
(2)\end{array}$ & $\begin{array}{c}\text { Decl. } \\
(\mathrm{J} 2000.0) \\
(3)\end{array}$ & $\begin{array}{l}\text { ID } \\
(4)\end{array}$ & $\begin{array}{l}\text { Group } \\
\text { (5) }\end{array}$ & $\begin{array}{c}V_{r} \\
\left(\mathrm{~km} \mathrm{~s}^{-1}\right) \\
(6)\end{array}$ \\
\hline A........ & 170329.1 & 783753.7 & 410 & 2 & 17796.4 \\
\hline B... & 170302.4 & 783553.8 & 335 & 1 & 16303.0 \\
\hline C. & 170329.6 & 783953.0 & 414 & 2 & 17558.0 \\
\hline 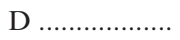 & 170447.8 & 783829.5 & 635 & 2 & 19094.2 \\
\hline Е & 170533.2 & 785116.3 & 752 & 3 & 19478.6 \\
\hline F1 .................. & 170217.9 & 784128.3 & & & \\
\hline 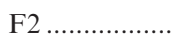 & 170614.5 & 783941.0 & & & \\
\hline F3 ................... & 170651.5 & 784106.3 & 946 & 2 & 16866.5 \\
\hline G .................. & 170343.0 & 785003.3 & 486 & 2 & 17115.2 \\
\hline $\mathrm{H}$ & 170231.3 & 784237.8 & & & \\
\hline I $\ldots \ldots \ldots \ldots \ldots \ldots \ldots \ldots \ldots \ldots \ldots$ & 170051.8 & 784119.8 & 88 & 2 & 17325.0 \\
\hline $\mathrm{J} \ldots \ldots \ldots \ldots \ldots \ldots \ldots \ldots \ldots \ldots$ & 170111.9 & 784326.5 & 116 & 1 & 16122.9 \\
\hline ............ & 170218.3 & 784602.2 & 233 & 3 & 19643.0 \\
\hline L.. & 170250.3 & 783148.4 & & & \\
\hline M ................... & 170929.3 & 784138.6 & & & \\
\hline $\mathrm{N}$ & 170146.4 & 780000.1 & & & \\
\hline O.................... & 170514.4 & 783017.8 & 706 & & \\
\hline $\mathrm{P} \ldots \ldots \ldots \ldots \ldots \ldots \ldots \ldots \ldots$ & 170103.8 & 783004.7 & 111 & & \\
\hline Q $\ldots \ldots \ldots \ldots \ldots \ldots \ldots \ldots \ldots \ldots$ & 170322.2 & 782505.5 & & & \\
\hline $\mathrm{R} \ldots \ldots \ldots \ldots \ldots \ldots$ & 165849.0 & 783721.0 & & & \\
\hline S ..................... & 165926.4 & 784114.9 & & & \\
\hline Т ..................... & 170356.6 & 775812.5 & & & \\
\hline $\mathrm{U} \ldots \ldots \ldots \ldots \ldots \ldots$ & 170915.2 & 782817.2 & & & \\
\hline V & 170019.9 & 782805.8 & & & \\
\hline $\mathrm{W} \ldots \ldots \ldots \ldots \ldots \ldots$ & 170947.1 & 785110.6 & & & \\
\hline $\mathrm{X} \ldots \ldots \ldots \ldots \ldots \ldots \ldots \ldots \ldots \ldots$ & 170728.2 & 784722.2 & & & \\
\hline Y ...................... & 170918.5 & 782902.6 & & & \\
\hline 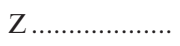 & 170353.3 & 784031.3 & & & \\
\hline AA ................... & 170832.4 & 782953.1 & & & \\
\hline 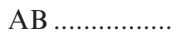 & 170404.2 & 782330.0 & & & \\
\hline $\mathrm{AC} \ldots \ldots \ldots \ldots \ldots$ & 170246.3 & 785145.5 & & & \\
\hline AD $\ldots \ldots \ldots \ldots \ldots$ & 165818.1 & 782933.8 & & & \\
\hline $\mathrm{AE} \ldots \ldots \ldots \ldots \ldots$ & 165904.0 & 784534.5 & & & \\
\hline $\mathrm{AF} \ldots \ldots \ldots \ldots \ldots$ & 165758.1 & 783615.2 & & & \\
\hline
\end{tabular}




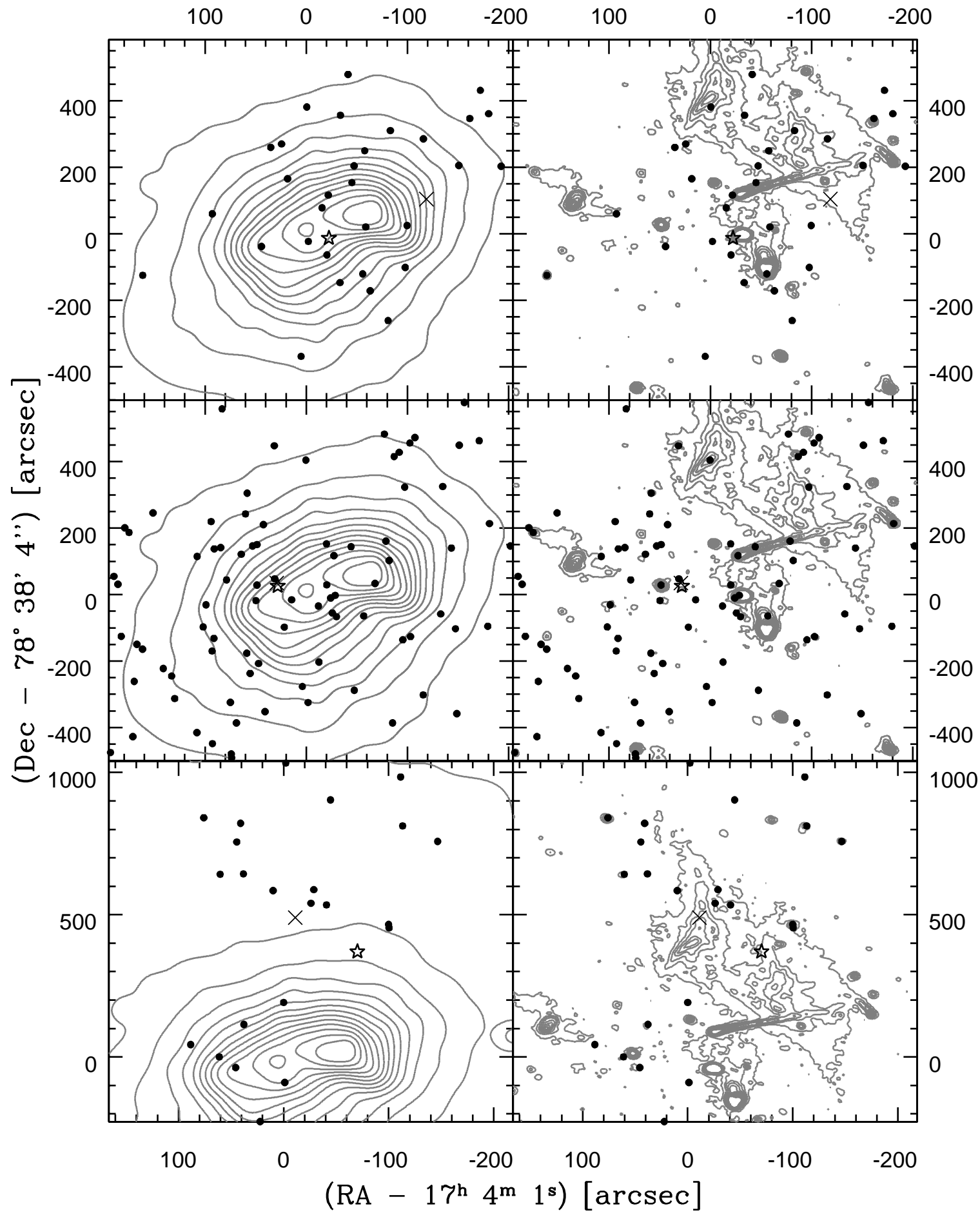

FIG. 12.-Positions of galaxies for groups 1,2, and 3 along with the X-ray (left panels) and radio contours (right panels). The top panels show the galaxies associated with group 1, the middle panels show galaxies associated with group 2 , and the bottom panels show the galaxies associated with group 3 .

gives the feature identification from Röttgering et al. (1994) and Bridle et al. (1979). Columns (2) and (3) give the R.A. and decl., and column (4) gives the optical counterpart if one is known. The identification number used in column (4) corresponds with the galaxy ID numbers used in Table 3. Column (5) gives the group membership of the optical counterpart if one is known, and column (6) gives the measured radial velocity of the galaxy.

There are a number of striking features in the radio map of A2256 that can be easily separated into the effects from the interaction between groups 1 and 2 versus the interaction between groups 2 and 3 . We shall discuss these two feature sets in turn. To facilitate the discussion, Figure 13 shows the positions of the radio peaks superimposed on the radio contours from Röttgering et al. (1994). The positions adopted are those given in Röttgering et al. (1994).

\subsubsection{Head-Tail Radio Sources A and B}

We interpret the combined radio feature formed from peaks $\mathrm{A}$ and $\mathrm{B}$ as the product of the merger event between 


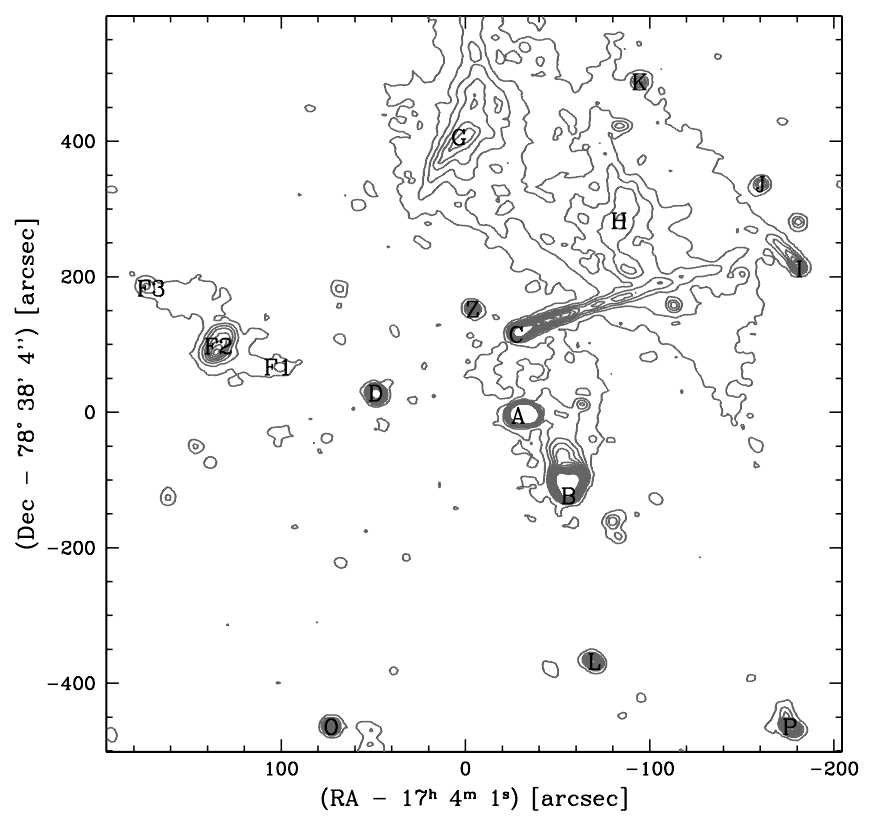

FIG. 13.-Positions of the radio peaks along with the radio contour map from Röttgering et al. (1994) in the central region of A2256, with the same naming convention as used in Bridle et al. (1979) and Röttgering et al. (1994) for simplicity. See Table 10 for celestial coordinates and optical counterparts.

groups 1 and 2. Table 10 indicates that galaxies associated with group 2 are the optical counterparts to peak A, and galaxies associated with group 1 are the counterparts to peak B. In our interpretation, group 1 is infalling radially from behind onto group 2, from the northwest, with a position angle consistent with the value given by Roëttiger et al. (1993). The radio feature A is most likely associated with the high-speed interaction between galaxies 410 and 420 of group 2 . Radio peak B is indisputably associated with galaxy 335 of group 1 . This feature is one of four known headtail sources. In most models a head-tail source is the trail of a radioactive galaxy that moves at high speeds relative to the intracluster medium. According to our model, source B is the interaction of galaxy 335 with the intracluster medium of group 2 as the two clusters merge.

\subsubsection{Diffuse Radio Emission Sources $G$ and $H$}

The diffuse emission feature marked by peaks $\mathrm{G}$ and $\mathrm{H}$ was originally interpreted as a shock feature between the merging groups 1 and 2 by Enßlin et al. (1998). However, we feel that this feature provides strong evidence that group 3 is bound and infalling on group 2 .

In support of this interpretation, there is a strong correlation between the location of the galaxies associated with group 3 as determined from the EM algorithm and the extended radio feature (see Fig. 12). The shape and position angle of the galaxy distribution closely follow the contour lines of the G-H radio feature. Each galaxy associated with peaks directly related to the extended radio feature is a member of either group 2 or group 3 with the central dominant galaxy associated with group 3 positioned central to the $\mathrm{G}-\mathrm{H}$ radio feature. The only exception is peak $\mathrm{J}$, which is not directly related to the $\mathrm{G}-\mathrm{H}$ radio halo but is associated with a cluster 1 member.
Röttgering et al. (1994) show a strong correlation between the X-ray contours and the northwest edge of the $\mathrm{G}-\mathrm{H}$ radio feature. Given the mass of group 3, the expected $\mathrm{X}$-ray thermal bremsstrahlung luminosity will be in the range of $10^{41}-10^{42} \mathrm{ergs} \mathrm{s}^{-1}$. Furthermore, computer simulations of particles accelerated by shocks formed between merging clusters of galaxies (Berrington, Dermer, \& Sturner 2001) show that the expected X-ray luminosity from the nonthermal particles accelerated by first-order Fermi in the $2-10 \mathrm{keV}$ energy range is $\sim 10^{43} \mathrm{ergs} \mathrm{s}^{-1}$ at peak luminosity. It is challenging to distinguish between nonthermal X-ray emission from the shock front and thermal bremsstrahlung emission from group 3 given the noise level of the ROSAT imaging. Emission from the shock front should dominate and more readily manifest itself in the ROSAT data as supported by the analysis performed by Röttgering et al. (1994). More recent observations by the Chandra X-Ray Observatory (Sun et al. 2002) indicate an X-ray excess in the region of group 3 and the $\mathrm{G}-\mathrm{H}$ radio feature.

\subsubsection{Head-Tail Radio Sources C and I}

Source $\mathrm{C}$ is unresolved laterally but is approximately 500 $\mathrm{kpc}$ in length with a kink in the tail $380 \mathrm{kpc}$ distant from the source (Röttgering et al. 1994). Galaxy 414, the radio source $\mathrm{C}$ optical counterpart, is a member of group 2. Our interpretation is that the head-tail radio feature $\mathrm{C}$ has just passed through the shock front marked by the G-H radio feature. The kink marks the point where the tail pops through the shock front into the intergalactic medium of group 3 . The velocity of galaxy 414 deviates from the velocity centroid by only $\sim 108 \mathrm{~km} \mathrm{~s}^{-1}$. Therefore, the orbital motion of galaxy 414 with respect to group 2 is mostly tangential. Röttgering et al. (1994) performed an orbit calculation assuming that the tail traces the trajectory of the galaxy through the cluster. They find the shape of the tail to be consistent with a galaxy with initial transverse velocity in the range of 2000-3500 $\mathrm{km} \mathrm{s}^{-1}$.

The head-tail source $\mathrm{I}$ is associated with galaxy 88 in group 2. This optical counterpart is found $\sim 340 \mathrm{~km} \mathrm{~s}^{-1}$ blueward of the group 2 centroid. There are no galaxies associated with any obvious interaction with galaxy 88 . One possible interpretation is that it is a galaxy that has recently passed from the intergalactic medium of group 2 and entered the intergalactic medium of group 3. Supporting this idea, the tail of source I extends only to the diffuse emission nebula source of $\mathrm{G}$ and $\mathrm{H}$. The radio sources $\mathrm{C}$ and $\mathrm{I}$ along with sources $\mathrm{G}$ and $\mathrm{H}$ provide strong evidence for interaction between groups 2 and 3 (see $\S 4.1$ ).

\section{SUMMARY AND CONCLUSIONS}

We have presented the results of a radial velocity survey of the galaxy cluster A2256, carried out with the Hydra Multi-Object Spectrograph on the WIYN telescope, in which we obtained a total of 277 radial velocities of cluster members. In addition, we presented new photometry and astrometry of 861 cluster members within the central $19^{\prime} \times 19^{\prime}$ of the cluster, obtained from a $3 \times 3$ WIYN image mosaic in the $R$ band. By applying a number of statistical tests, we found strong evidence of statistically significant deviations from the expected single-Gaussian velocity distribution of a relaxed cluster. On application of the KMM software package, which is based on the EM algorithm for 
mixture modeling (McLachlan \& Basford 1988), we found a total of three subclusters, only two of which had been previously noted. This analysis used both velocity and spatial distribution data to identify the separate groups. We obtained virial mass estimates for each group and showed that the total of the group masses is less than half the inferred virial mass of the entire cluster, in support of the presence of substructure. We then applied the two-body orbit model of Beers et al. (1982), first to the two-body system defined by groups 1 and 2 alone and then to a system in which groups 1 and 2 were treated as a single body and group 3 was taken as the other body. This dynamical analysis strongly indicates that groups 1 and 2 are an infalling, bound system. However, it does not, in itself, fix the dynamical status of group 3 within the A2256 system.

To help determine whether group 3 is bound to the main cluster, we correlated our optical results with previous Xray and radio imaging of A2256. The X-ray surface brightness distribution of A2256 has two centrally located peaks (Briel et al. 1991). The galaxy groups 1 and 2, which we identified from the galaxy position and velocity distributions, are strongly correlated with the X-ray peaks, providing additional support for the merging subcluster interpretation. The optical data alone allow group 3 to be modeled as either bound, and thus in close proximity with the 1-2 system, or unbound, and thus not interacting with the 1-2 system. However, radio maps of A2256 (Röttgering et al. 1994) provide strong evidence for an interaction between groups 2 and 3 and thus clearly support the bound model. As discussed in $\S 4.4$, the diffuse radio emission feature is probably a shock front between the intracluster media of groups 2 and 3. In addition, the interaction of the head-tail sources $\mathrm{C}$ and I with the diffuse radio emission feature provides evidence that the two systems are interacting (see $\S$ 4.4.2).

In summary, our proposed model of A2256 consists of three bound subgroups that are undergoing merging. Groups 1 and 2 appear to be in an advanced stage of the merging process, near the time of the first close passage of the group centers. In this model, group 1 is located behind group 2 and is infalling from the northwest. Pinkney et al. (1996) suggest that the lines of center lie at approximately $45^{\circ}$ from the line of sight, which is consistent with the range allowed for bound orbits. According to our model, group 3 is located on the near side of the 1-2 system and is infalling from the north. Our two-body analysis indicates that the infall direction lies at an angle to the line of sight in the range $50^{\circ}-60^{\circ}$. The dynamics of the dominant group 2 are probably strongly influenced by the ongoing merger with group 1, but the 1-2 merger system may be largely unaffected by the presence of the much smaller group 3. Each group contains a central, dominant galaxy that, for groups 1 and 2, is located near the X-ray surface brightness peak. This is consistent with the prediction that the dominant galaxy of a group will quickly settle into the center of the local potential well (Bode et al. 1994). The greatest offset between the galaxy distribution centroid, the position of the central dominant galaxy, and the X-ray peak occurs in group 1, suggesting that the apparent merger event is strongly influencing the dynamics of this group.

As discussed by Henriksen (1999), there is some continuing controversy regarding the merger model for interpreting the X-ray observations of A2256. Thus, further X-ray observations of A2256 would help to distinguish between the cases of merging subclusters and clusters that are merely superimposed on the sky by projection (Henriksen 1999). Briel \& Henry (1994) suggest the presence of a complex temperature structure in the X-ray intensity distribution, but this is not strongly supported by observations made by ASCA (Markevitch 1996). Because of the high-velocity impact between groups 1 and 2, the presence of a shock front is likely and has been predicted by computer simulations (Roëttiger et al. 1993). A2256 is an excellent target for Chandra. Higher resolution X-ray images and better temperature maps of the A2256 field would greatly increase our understanding of the dynamics of the multiple merger events that appear to be shaping this system.

We thank Mike Pierce for many long and useful discussions concerning the data acquisition, reduction, and interpretation. We thank Ulrich Briel and J. Patrick Henry for the use of the ROSAT X-ray data in Figure 12. We thank Huub Röttgering and Ignas Snellen for the use of the VLA radio data in Figure 12. We also thank the Indiana University Astronomy Department for travel support to carry out the necessary observations at the WIYN telescope.
Abell, G. O. 1958, ApJS, 3, 211

Ashman, K. M., Bird, C. M., \& Zepf, S. E. 1994, AJ, 108, 2348

Beers, T. C., Flynn, K., \& Gebhardt, K. 1990, AJ, 100, 32

Beers, T. C., Geller, M. J., \& Huchra, J. P. 1982, ApJ, 257, 23

Berrington, R. C., Dermer, C. D., \& Sturner, S. J. 2001, in AIP Conf. Proc. 587, Accelerated Particles from Shocks Formed in Merging Clusters of Galaxies, ed. S. Ritz, N. Gehrels, \& C. R. Shrader (New York: AIP), 411 Bird, C. M. 1994, AJ, 107, 1637

Bird, C. M., \& Beers, T. C. 1993, AJ, 105, 1596

Bode, P., Berrington, R. C., Cohn, H. N., \& Lugger, P. M. 1994, ApJ, 433, 479

Bridle, A. H., Fomalont, E. B., Miley, G. K., \& Valentijn, E. A. 1979, A\&A, 80, 201

Briel, U. G., et al. 1991, A\&A, 246, L10

Briel, U. G., \& Henry, J. P. 1994, Nature, 372, 439

Burstein, D. \& Heiles, C. 1982, AJ, 87, 1165

Carter, D., \& Metcalfe, N. 1980, MNRAS, 191, 325

D'Agostino, R. B., \& Stephens, M. A. 1986, Goodness-of-Fit Techniques

(New York: Marcel Dekker)

Dressler, A. 1978, ApJ, 226, 55

Dressler, A., \& Shectman, S. A. 1988, AJ, 95, 985

Enßlin, T. A., Biermann, P. L., Klein, U., \& Kohle, S. 1998, A\&A, 332, 395

Faber, S., \& Dressler, A. 1977, AJ, 82, 187

Fabricant, D. G., Kent, S. M., \& Kurtz, M. J. 1989, ApJ, 336, 77

\section{REFERENCES}

Fabricant, D G., Rybicki, G., \& Gorenstein, P. 1984, ApJ, 286, 186

Fernie, J. D. 1983, PASP, 95, 782

Fitchett, M. 1988, MNRAS, 230, 161

Gebhardt, K., \& Beers, T. C. 1991, ApJ, 383, 72

Heisler, J., Tremaine, S., \& Bahcall, J. N. 1985, ApJ, 298, 8

Henriksen, M. 1999, ApJ, 511, 666

Iglewicz, B. 1983, Robust Scale Estimators and Confidence Intervals for Location, Understanding Robust and Exploratory Data Analysis (New York: Wiley)

Lee, K. L. 1979, J. Am. Stat. Assoc., 74, 708

Markevitch, M. 1996, ApJ, 465, L1

McLachlan, G. J., \& Bastford, K. E. 1988, Mixture Models: Interference and Applications to Clustering (New York: Marcel Dekker)

McLachlan, G. J., \& Krishnan, T. 1997, The EM Algorithm and Extensions (New York: Wiley)

Pearson, E. S., \& Hartley, H. O. 1962, Biometrika Tables for Statisticians (Cambridge: Cambridge Univ. Press)

Pence, W. 1976, ApJ, 203, 39

Pinkney, J., Roëttiger, K., Burns, J. O., \& Bird, C. M. 1996, ApJS, 104, 1

Postman, M., \& Lauer, T. R. 1995, ApJ, 440, 28

Rhee, G. F. R. N., van Haarlem, M. P., \& Katgert, P. 1991, A\&AS, 91, 513

Roëttiger, K., Burns, J., \& Loken, C. 1993, ApJ, 407, L53

Röttgering, H., Snellen, I., Miley, G., de Jong, J. P., Hanisch, R. J., \& Perley, R. 1994, ApJ, 436, 654 
Shapiro, S. S., \& Wilk, M. B. 1965, Biometrika, 52, 591

Stephens, M. A. 1986, Tests Based on EDF Statistics, Goodness-of-Fit Techniques, ed. R. B. D’Agostino \& M. A. Stephens (New York: Marcel Dekker)

Sun, M., Murray, S. S., Markevitch, M., \& Vikhlinin, A. 2002, ApJ, 565, 867
West, M. J., \& Bothun, G. D. 1990, ApJ, 350, 36

Yahil, A., \& Vidal, N. D. 1977, ApJ, 214, 347

Zabludoff, A. I., Geller, M. J., Huchra, J. P., \& Vogeley, M. S. 1993, AJ, 106,1273 